\title{
Simulation Guided 3D Nanomanufacturing via Focused Electron Beam Induced Deposition
}

Jason D. Fowlkes ${ }^{a, b}$, Robert Winkler ${ }^{c}$, Brett B. Lewis ${ }^{b}$, Michael G. Stanford ${ }^{b}$ Harald Plank $^{c, d}$ and Philip D. Rack ${ }^{a, b}$

a Nanofabrication Research Laboratory, Center for Nanophase Materials Sciences, Oak Ridge National Laboratory, Oak Ridge, Tennessee 37831, United States

$b$ Materials Science and Engineering Department, The University of Tennessee, Knoxville, Tennessee 37996, United States

${ }^{c}$ Graz Centre for Electron Microscopy, Steyrergasse 17, 8010 Graz, Austria.

${ }^{d}$ Institute for Electron Microscopy and Nanoanalysis, Graz University of Technology, Steyrergasse 17, 8010 Graz, Austria

\section{Supplement 1}

\section{$3 \mathrm{D}$ rendering}

Surface voxels are rendered as shaded cubes in the 3D object images. Precursor coverage at each surface voxel is indicated by color. The precursor coverage is normalized to the maximum coverage. As a result, shadowing effects linked to the directed component of the impinging precursor flux are observed. The gas flux vector (grey) shows the directed component of the precursor flux. The gas flux vector is aimed at the beam impact region. Directly above the beam impact point the Gaussian nature of the beam is indicated by a green patch object. The z-component indicates the relative intensity of the beam, in its own z-coordinate system, while the FWHM of the beam profile accurately reflects the beam shape in the coordinate system of the deposit. The substrate lies in the $x-y$ plane and the out-of-plane coordinate is $z$. Information to render the deposit is saved following each dwell time in the beam pattern.

Surface voxels lying in the substrate plane also exhibit transparency. Beyond the boundary, surface voxels are not rendered to (1) save rendering time and (2) provide contrast with the white background for the upper portions of the 3D object.

During each dwell, a defined number of electron trajectories are sampled to provide a visualization of the interaction of individual electron trajectories with the deposit. These are shown as the green trajectories. The surface voxels are given a slight transparency value within a small sphere $(\mathrm{r}=25 \mathrm{~nm})$ centered at the beam impact point so that the partial penetration of the primary beam may be observed.

\section{S1_FEBID_Segment.wmv}




\section{Supplement 2}

While the cutoff energy of $100 \mathrm{eV}$ is above the energy necessary to create a secondary electron $(\varepsilon=73 \mathrm{eV})$, the primary electron position at the end of its trajectory is typically very deep in the solid $(\sim 2.2 \mu \mathrm{m})$ for the an electron acceleration voltage of $30,000 \mathrm{eV}$ as shown in the figure S2.1;

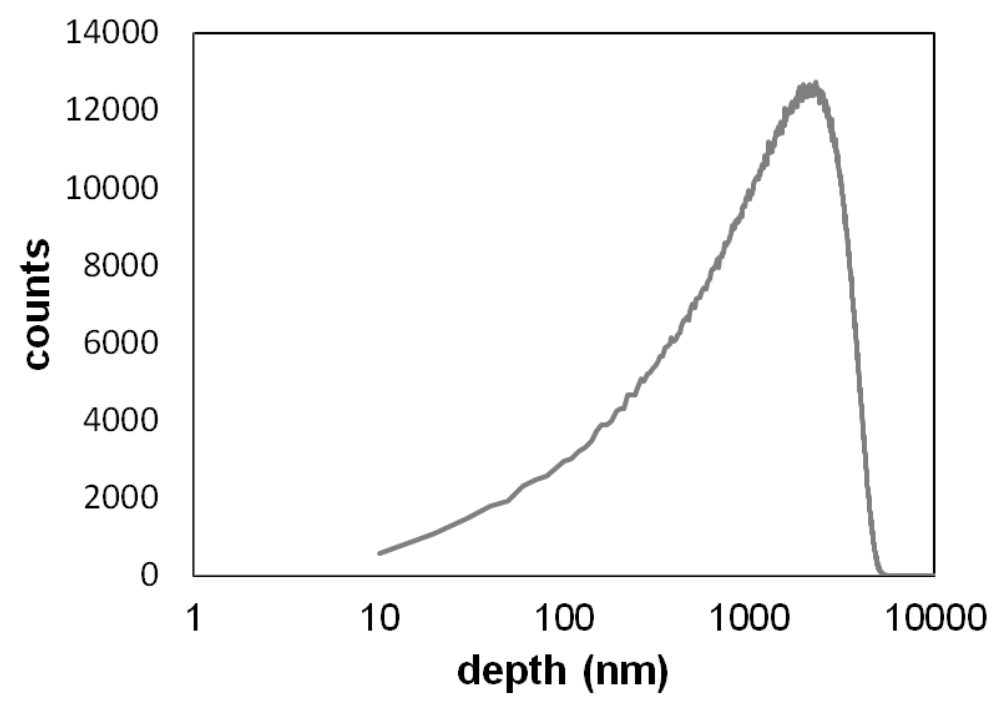

Figure S2.1 A histogram of the depth at which the primary electron energy $(30 \mathrm{keV})$ decays to the cutoff value of $(100 \mathrm{eV})$ in the $\mathrm{PtC}_{5}$ solid. The histogram was constructed using five million electron trajectories. It was assumed that the electron is reabsorbed by the solid at $100 \mathrm{eV}$. A semi-infinite bulk substrate was used consisting of flat $\mathrm{PtC}_{5}$ where the vacuum interface was located at (depth $=0 \mathrm{~nm})$ and the bulk extended into the region for $($ depth $>0)$. (depth $<0)$ was the vacuum phase. The bin size of the histogram is $10 \mathrm{~nm}$.

Thus, secondary electrons created at the end of primary trajectories contributed negligibly to the surface emitted SE distribution because of the small inelastic mean free path of the SE $(\lambda=$ $2.5 \mathrm{~nm}$ ). In addition, the total number of secondary electrons created between the incident energy and the cutoff energy for a single primary electron is;

$$
\frac{(30,000-100) \mathrm{eV}}{73.1 \frac{\mathrm{eV}}{\mathrm{SE}}}=\frac{29,900 \mathrm{eV}}{73.1 \frac{\mathrm{eV}}{\mathrm{SE}}}=409.0 \mathrm{SE}
$$

while only;

$$
\frac{100 \mathrm{eV}}{73.1 \frac{\mathrm{eV}}{\mathrm{SE}}}=1.37 \mathrm{SE}
$$

would be generated if the cutoff energy were extended to $0 \mathrm{eV}$ from $100 \mathrm{eV}$. This is only $0.3 \%$ of the total number of secondary electrons generated per primary electron. 


\section{Supplement 3}

The following slides may also be viewed in the file, "Supplement 3 (electron trajectory mapping.pdf".

\section{Supplement 3}

Schematic description of the electron trajectory mapping translated from the Monte Carlo simulation to the continuum solver 
Each primary electron trajectory is launched from a position that is buried within an infinite solid (figure S3.1). The solid has the properties of the material to be deposited, e.g., atomic number, atomic weight and density. Moreover, each trajectory is initiated from a common starting position (green data point indicated with white border) and with a propagation vector directed along the $-z$ direction. An elastic scattering event is indicated by each green data point. A large population (tens-of-thousands) of individual trajectories are generated although only a single trajectory is shown here for demonstration. Later, these trajectories are randomly called during execution of the continuum simulation in order to construct the $3 \mathrm{D}$ electron-solid interaction profile, for each unique beam impact position. As the following slides will demonstrate, the use of the infinite solid approach in the Monte Carlo simulation makes it possible to map the 3D electron-solid interaction profile for a 3D object of any shape. An example object is shown in (figure S3.2) with the center of the beam impact region (BIR) indicated by the light red line. The real beam shape is best described by a Gaussian profile (red curve) where (I) is the beam current and ( $\mathrm{x}$ ) is one of three Cartesian coordinates.

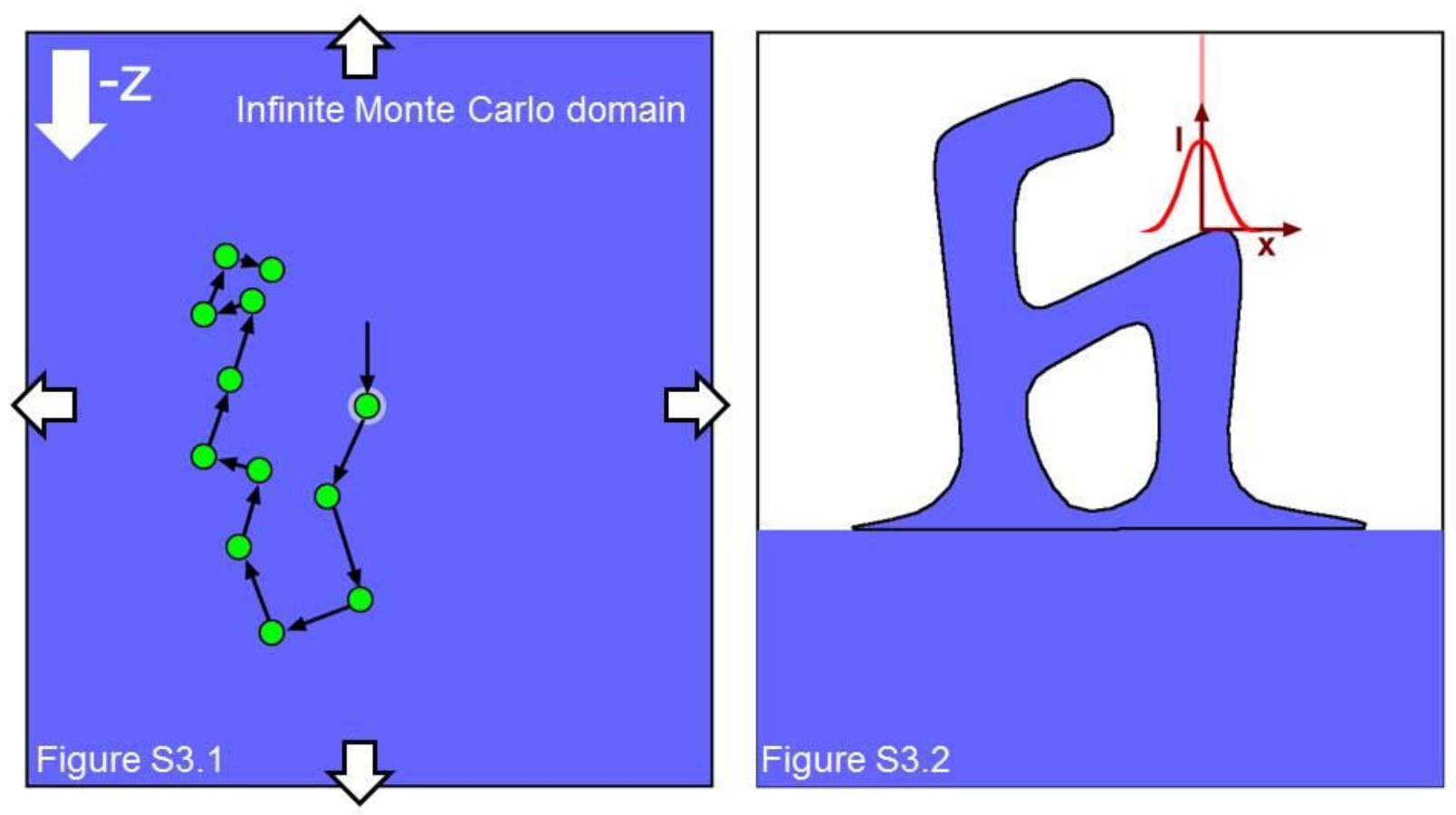


The algorithm for the construction of the 3D electron-solid interaction profile is triggered in response to a primary electron beam displacement. A primary electron trajectory is randomly selected. The trajectory is translated to the centerline of the beam impact region (large, yellow arrow, figure S3.3).

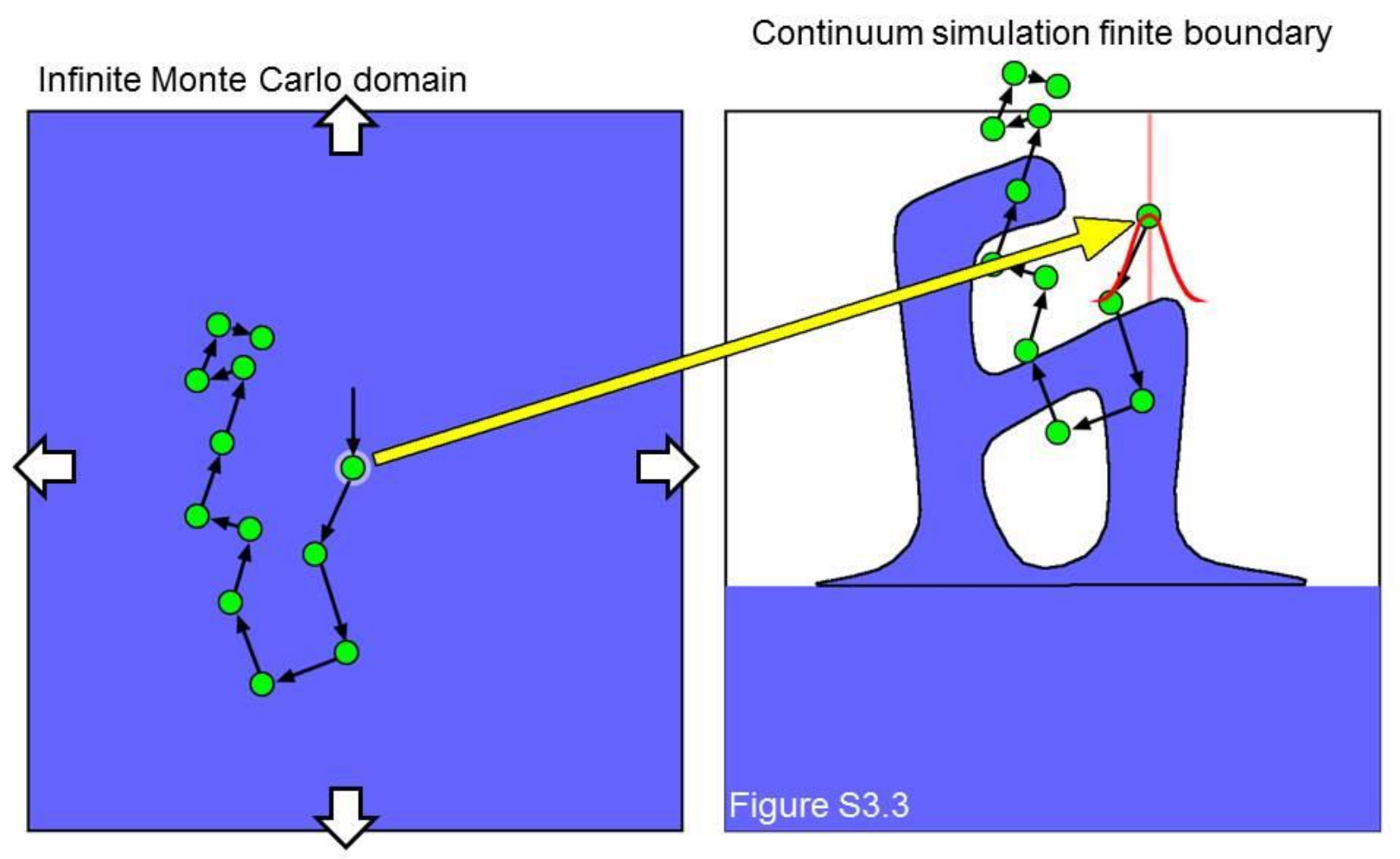


Next, random number sampling weighted to the Gaussian distribution, is used to determine a refined primary electron impact point (yellow arrows in figure S3.4). The surface position of the 3D deposit at the beam impact $(\mathrm{x}, \mathrm{y})$ region is aligned in the $\mathrm{z}$ coordinate with the first elastic scattering event of the trajectory. The previous position of the trajectory, taken from the last viewgraph, is shown in transparency for reference.

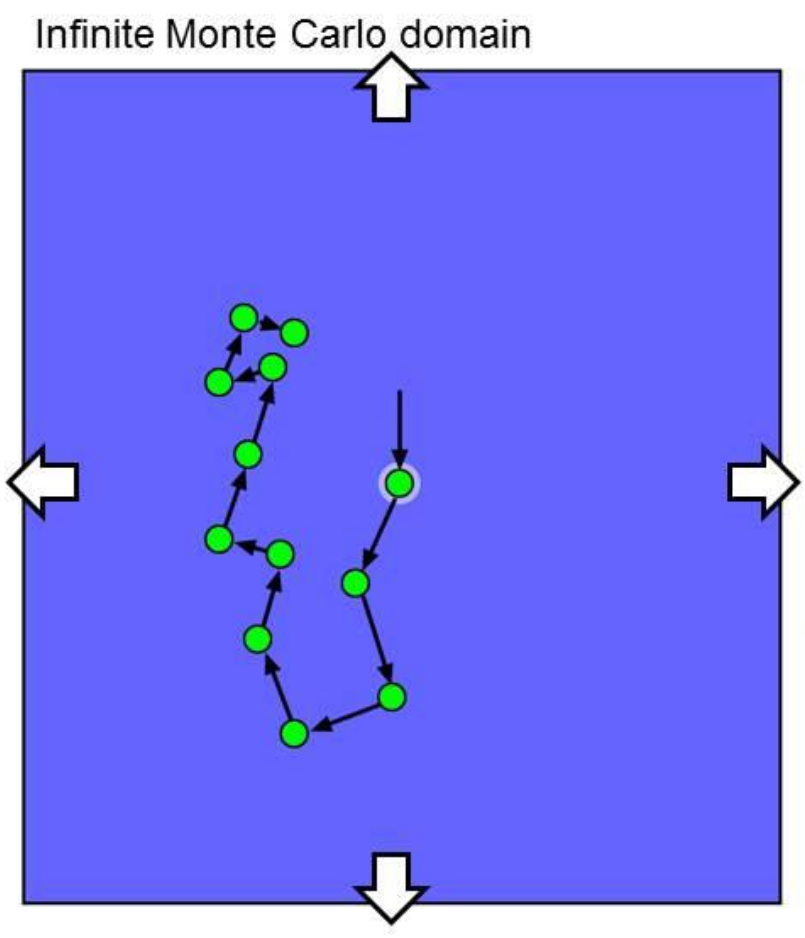

Continuum simulation finite boundary

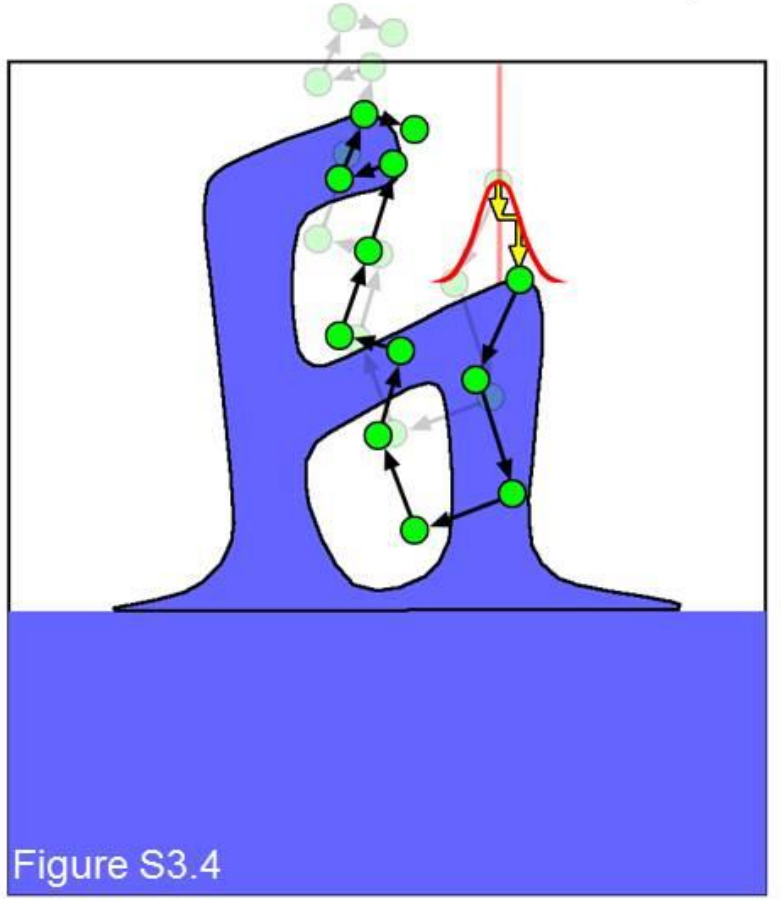


The next step is the adaption of the trajectory (figure S3.5) to the 3D deposit by (1) accounting for vacant space, e.g., translation through the vapor phase or (2) escape from the continuum simulation boundary. This process is shown on the following viewgraph figure S3.6.

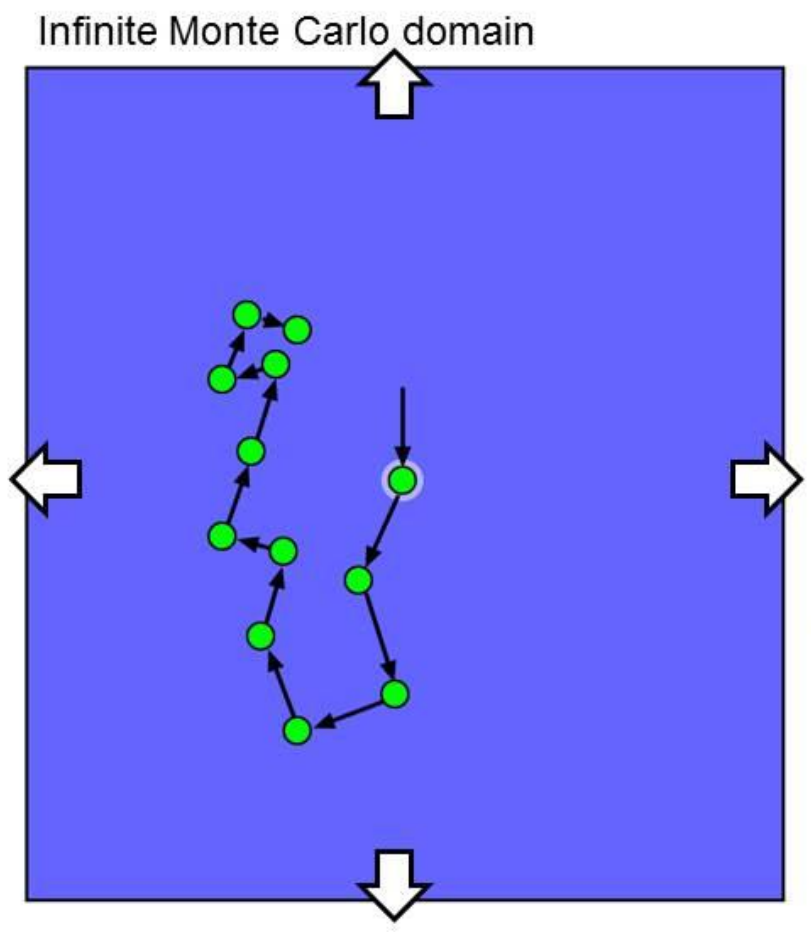

\section{Continuum simulation finite boundary}

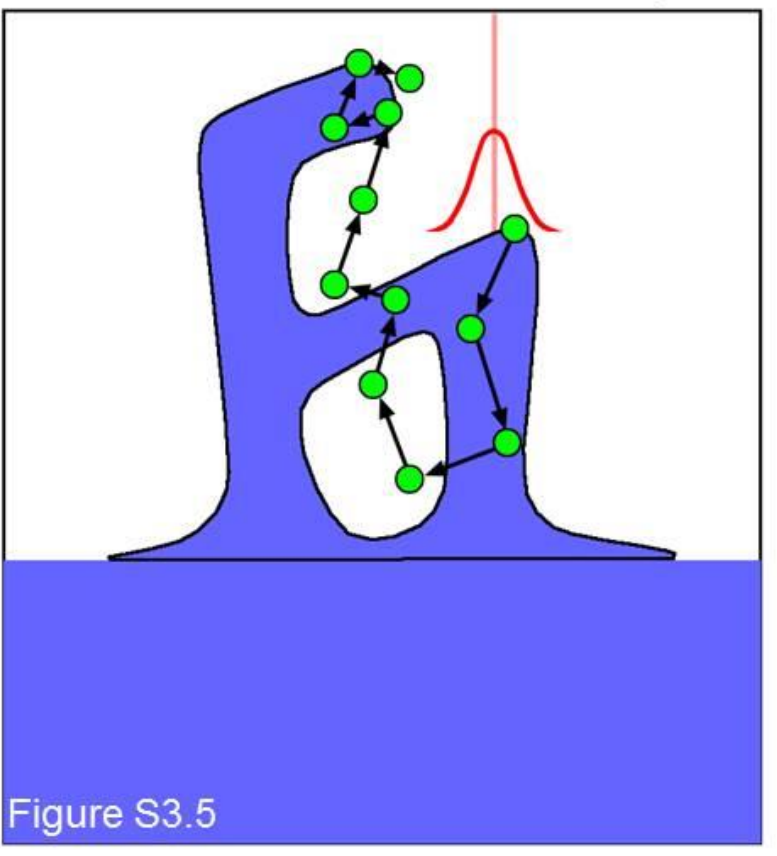


Event (\#1) (figure S3.6) indicates a pure translation across a region of vapor phase space while event (\#2) represents both an escape from the dense solid and intersection with the continuum simulation boundary. Intersection with the simulation boundary terminates the tracking of the trajectory. This completes adaption of the trajectory to the deposit.

\section{Infinite Monte Carlo domain}

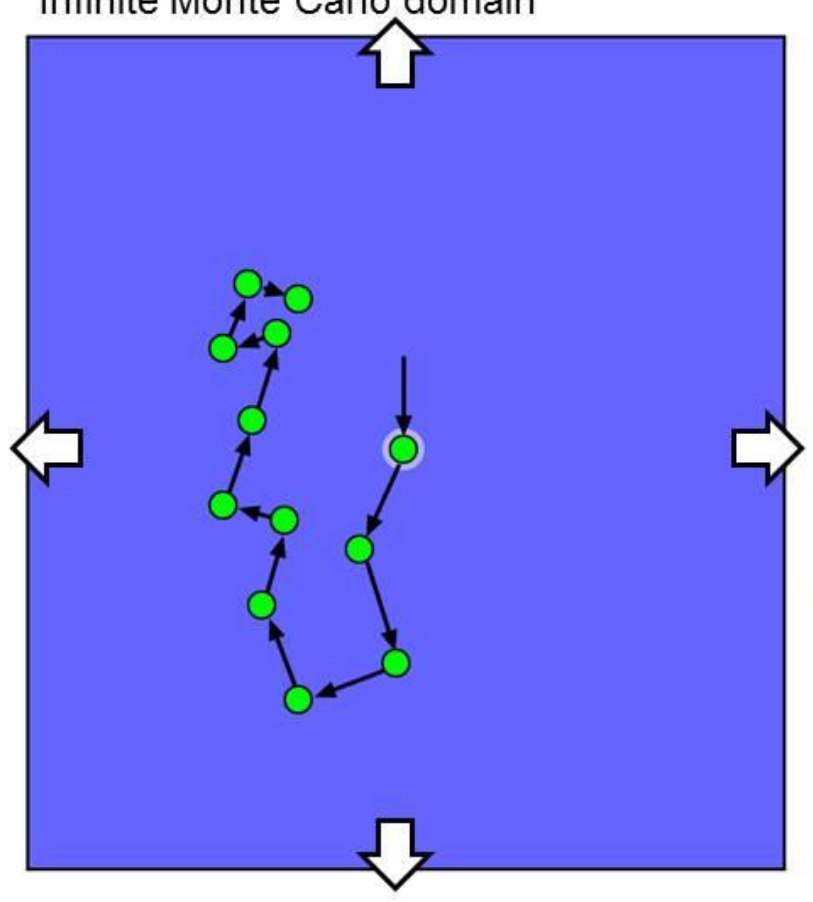

Continuum simulation finite boundary

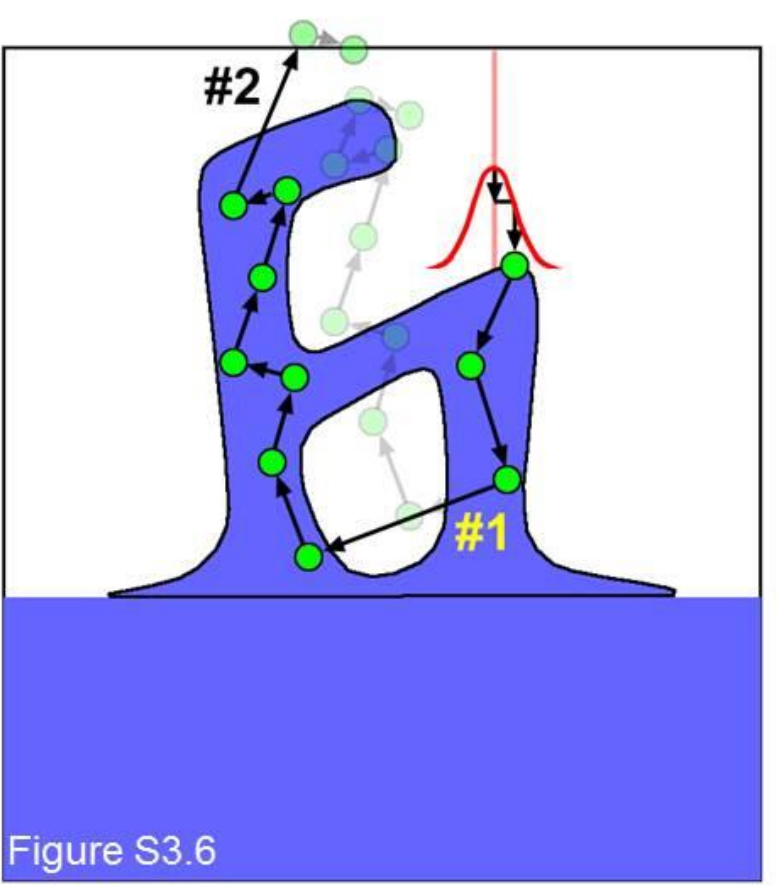


Here is shown the trajectory before (left) and after (figure S3.7) adaption.

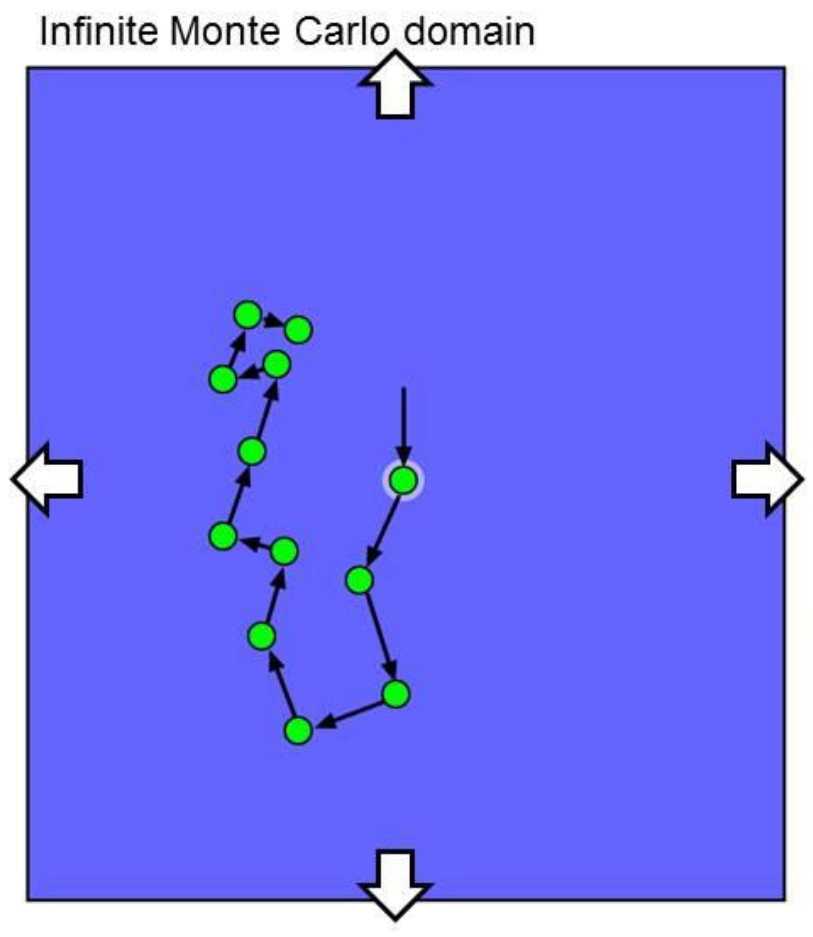

Continuum simulation finite boundary

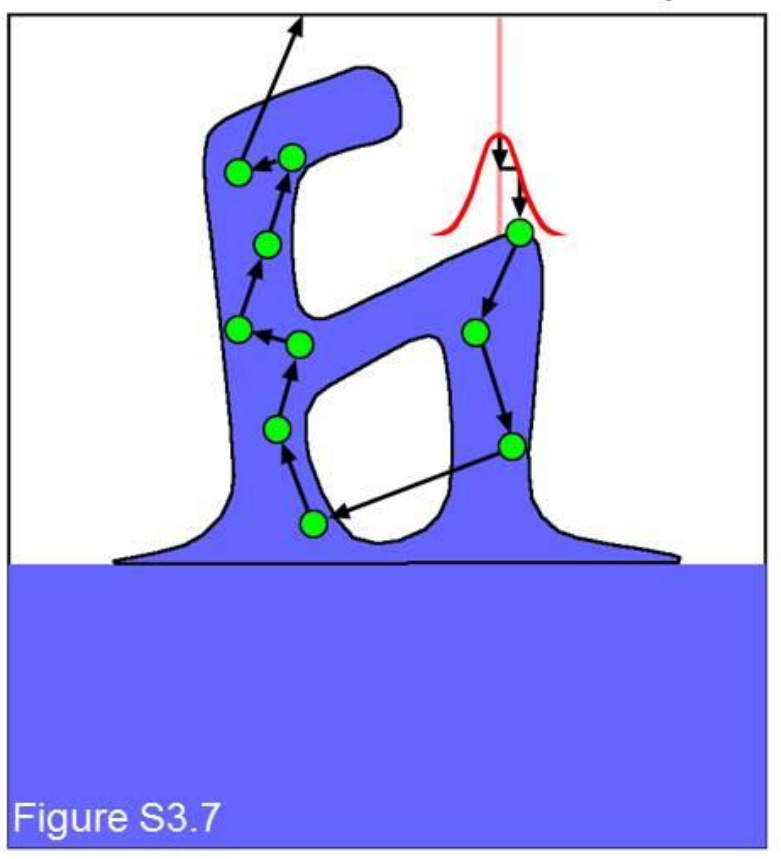


Secondary electron creation from the adapted trajectory follows as the next step. Each linear segment of the primary electron trajectory connects the $\left(n^{\text {th }}\right)$ and $\left(n+1^{\text {th }}\right)$ elastic scattering events. These segments are divided into sub-voxel size increments (figure S3.8) in order to allocate inelastic energy deposition into the solid. The continuous slowing down approximation predicts the rate of energy loss for each segment (dE/ds). Inelastic energy is distributed to each green data point according to $\Delta \mathrm{E}=$ $(\mathrm{dE} / \mathrm{ds}) \Delta \mathrm{s}$ where $(\Delta \mathrm{s})$ is the distance between the green data points. This inelastic energy is converted into a quantity of secondary electrons by dividing $(\Delta \mathrm{E})$ by the energy required to create a secondary electron $(\varepsilon)$. The emission of secondary electrons is described on the next viewgraph, figure S3.9.

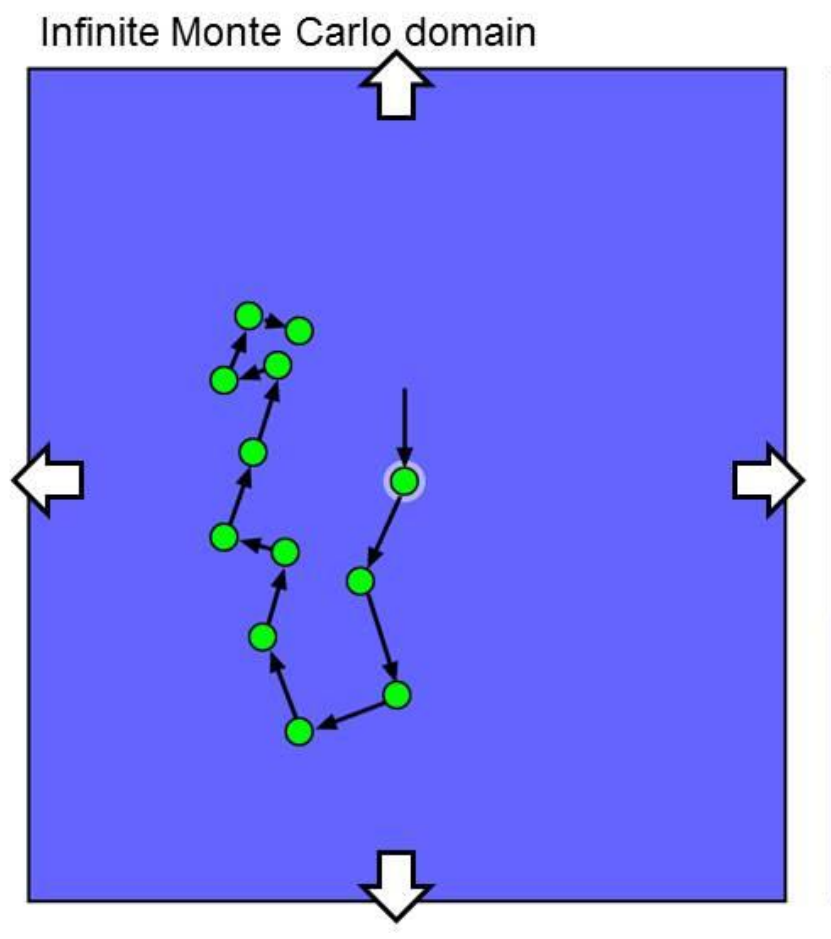

\section{Continuum simulation finite boundary}

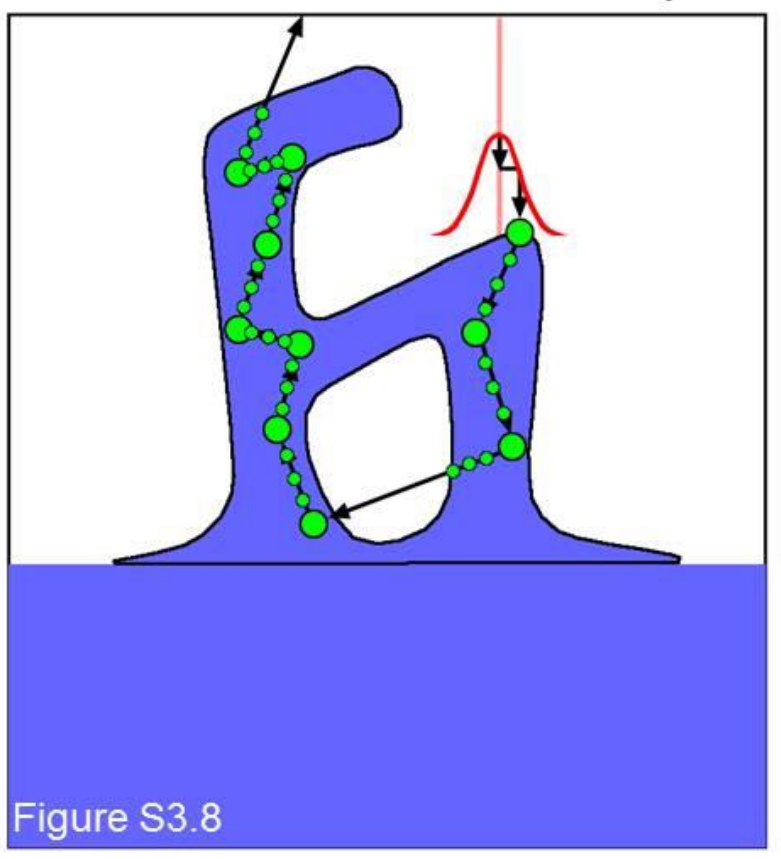


Secondary electrons (SE) are emitted in an isotropic manner from each green data point along the primary electron trajectory. The radius of emission is equal to the mean free path of the secondary electron $(\lambda)$ which is an input parameter for the simulation. Random point picking from a spherical surface is used to determine the trajectory of the (SE). The hot color map below (figure S3.9) indicates qualitatively the SE emission profile for the primary electron trajectory. The highest probability of finding an SE is indicated by red shaded regions in the color map while yellow shaded regions indicate a lower probability of a finding an SE.

\section{Infinite Monte Carlo domain}

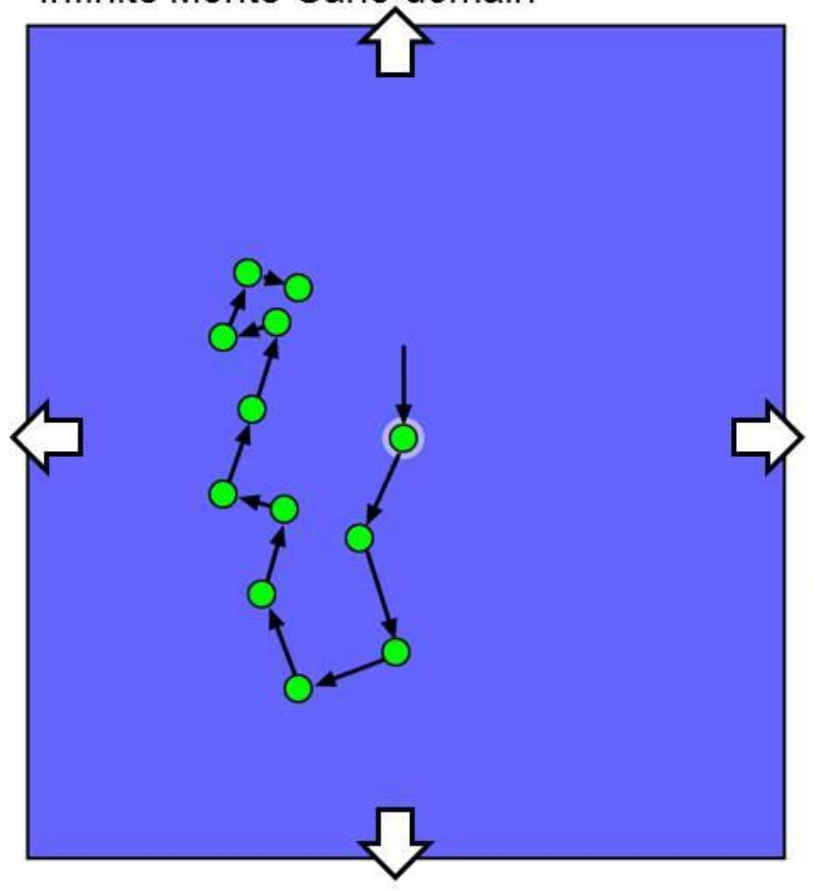

\section{Continuum simulation finite boundary}

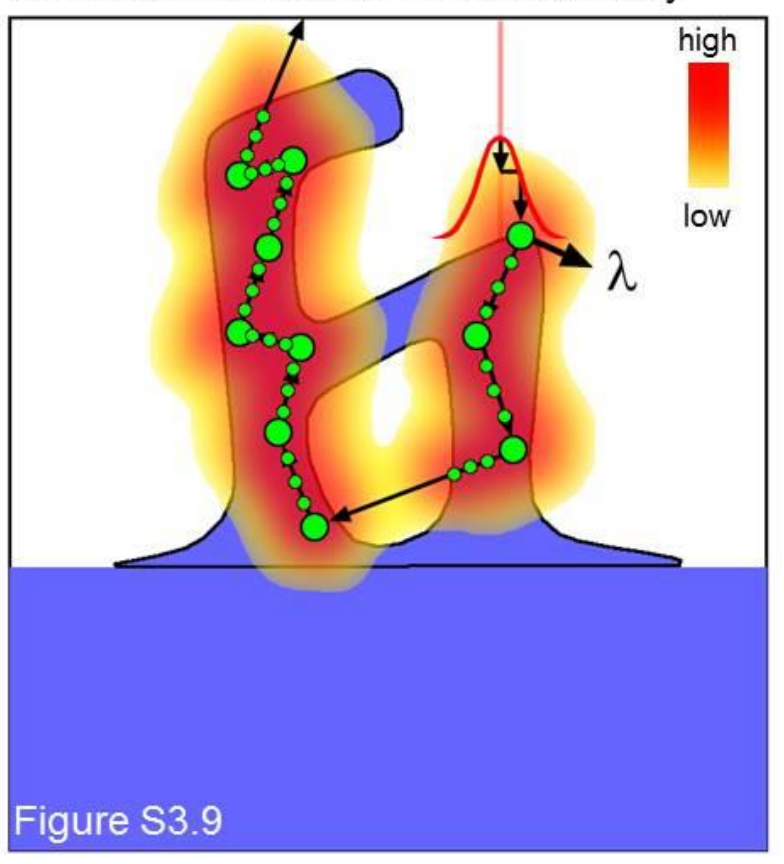


The continuum simulation domain consists of a 3D Cartesian domain of voxels. The surface voxels that surround the deposit (mesh network in figure S3.10) represent the voxels where the differential equation describing precursor surface coverage, precursor electron impact dissociation and surface diffusion will be calculated. Each SE emitted is tested to determine intersection events with the surface voxel population. In the event that an intersection is detected, the SE is associated with that surface voxel. SE accumulation is repeated for each sub-division per trajectory, and for all trajectories, leading to the construction of a 3D SE emission profile. The SE emission profile serves as a multiplication factor in the electron dissociation term in the continuum simulation (equation 3, main text) and serves as the link between the Monte Carlo simulation and the continuum simulation.

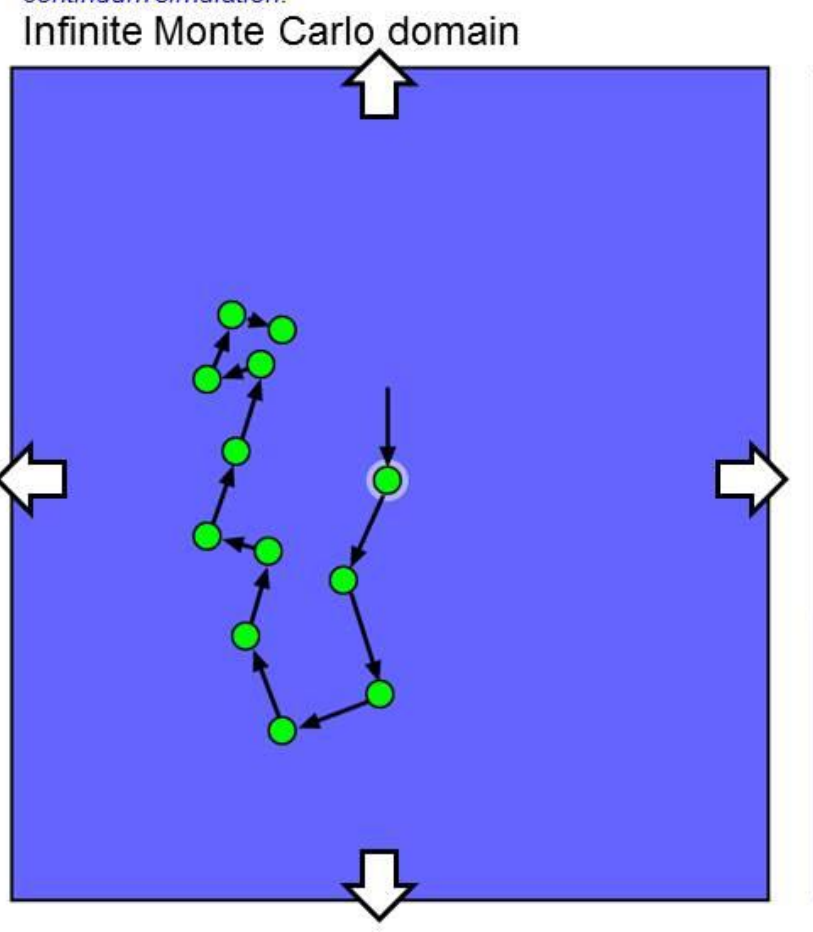

\section{Continuum simulation finite boundary}

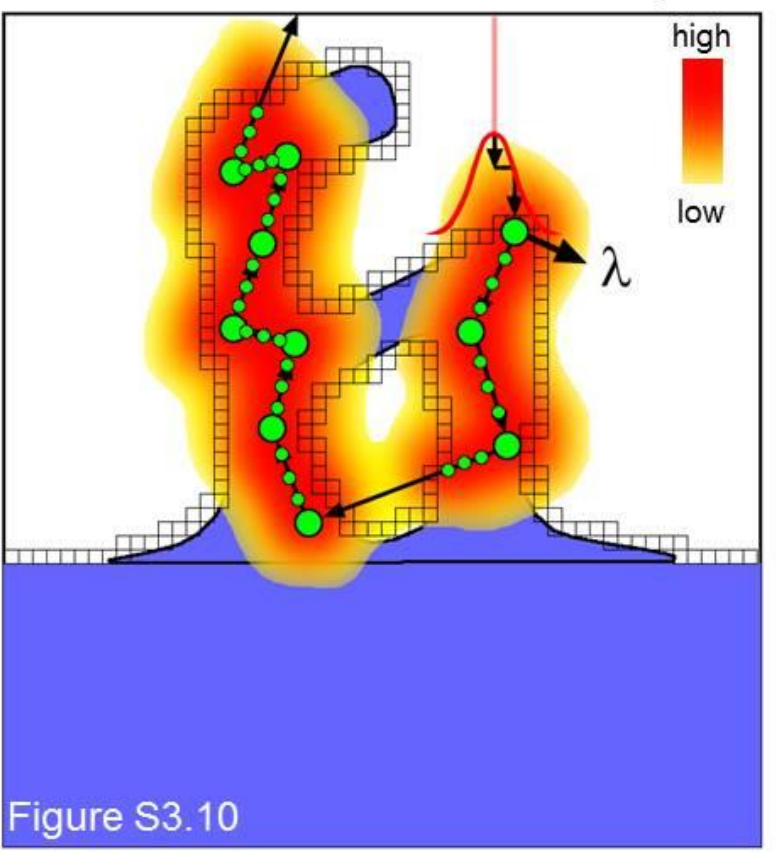


The SE surface density populating the surface voxel matrix is shown in figure S3.11 for the current beam position (light red line).

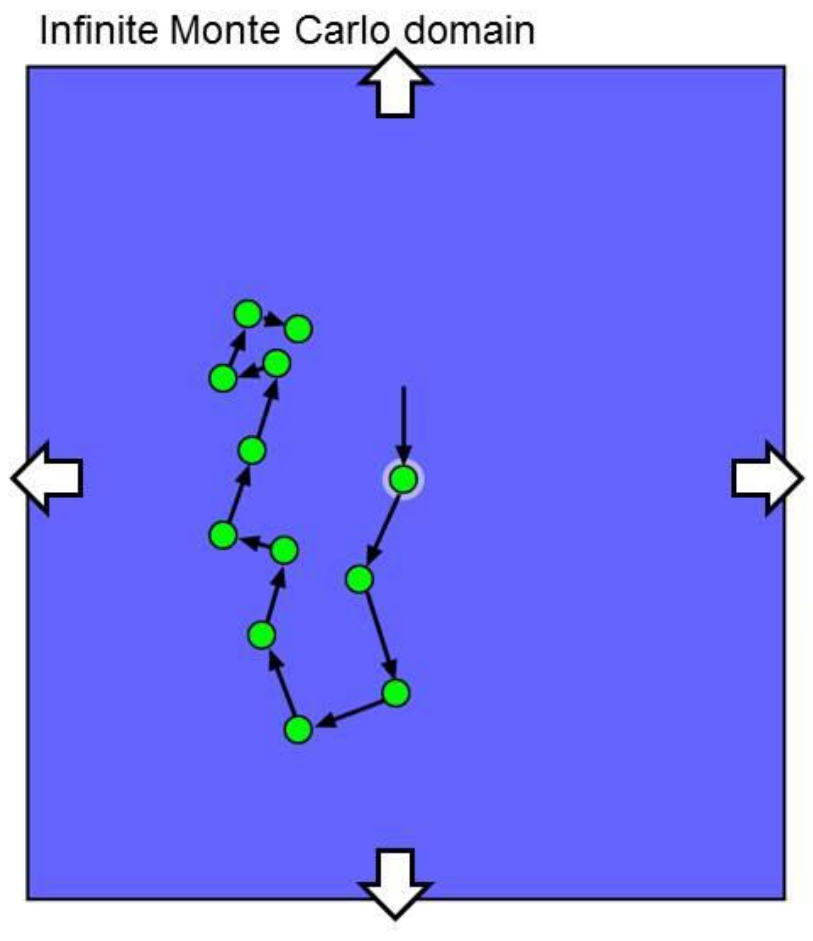

Continuum simulation finite boundary

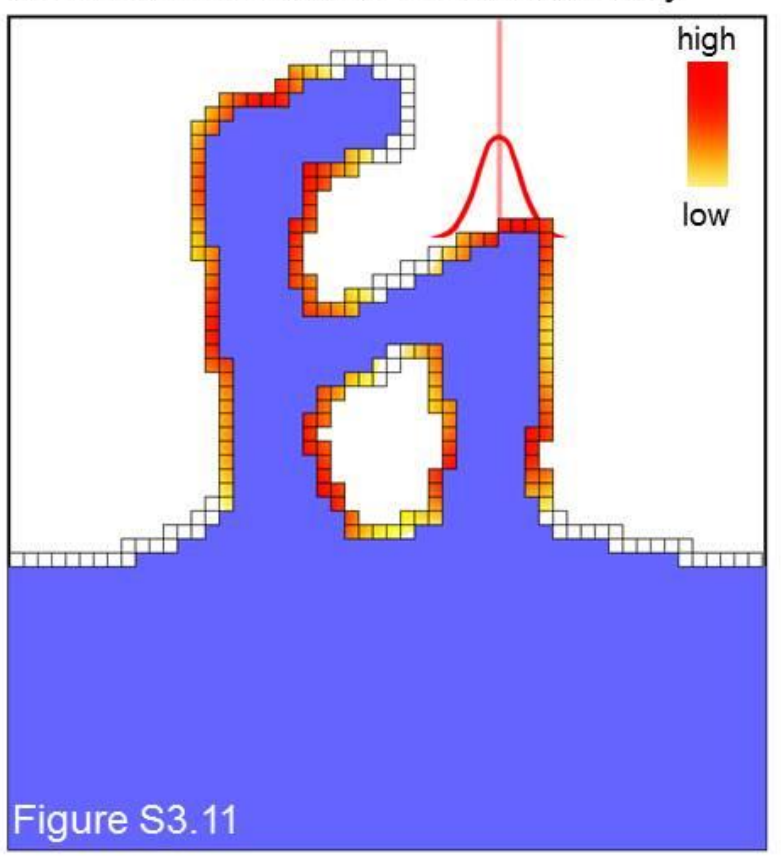




\section{Supplement 4}

The number of sampled electron trajectories per dwell $\left(\mathrm{N}_{\mathrm{d}}\right)$ was set to be much less than the actual number derived from the beam current $\left(\mathrm{i}_{\mathrm{b}}\right)$ and dwell time $\left(\tau_{\mathrm{d}}\right)$;

$$
N_{d}<<\frac{i_{b} \tau_{d}}{q} \quad[1]
$$

in order to accelerate simulation process time. This was possible because the electron spatial distribution could be approximated by a small number of electrons, for the voxel size of the $8 \mathrm{~nm}$ used, and the distribution simply multiplied by a scaling factor to represent the total current. The artifact of this procedure is the introduction of noise in the deposited energy profile spanning the beam interaction volume. This effect can best be seen in the spatial distribution of the primary beam impact as shown in figure S4.1(a). The radial electron spatial distribution profiles for different scaling factors are demonstrated where the yellow curve simulates, for example, 315,000 electrons and the other curves are smaller sample sizes which are 4, 40, and 40,000 times fewer electrons and normalized by the scaling factor. Clearly if the sample size is too small, the graph becomes too noisy. However, this effect is damped due to the relatively large $(8 \mathrm{~nm})$ voxel size used in the simulation. Figures $S 4.1(\mathrm{~b}-\mathrm{e})$ illustrate the effective spot size relative to a single voxel where the color map is a base 10 logarithm of the electron histogram is shown in the $(x-y)$ plane over the beam impact region. The bin size in these examples is $0.5 \mathrm{~nm}$. The voxel size $(8 \mathrm{~nm})$ of the simulation has been overlaid with the center of the beam impact region for comparison. Lastly, simulated FEBID results are shown in figure S4.2(a-c) using the complementary beam profiles presented in figure $S 4.1(b-d)$. 

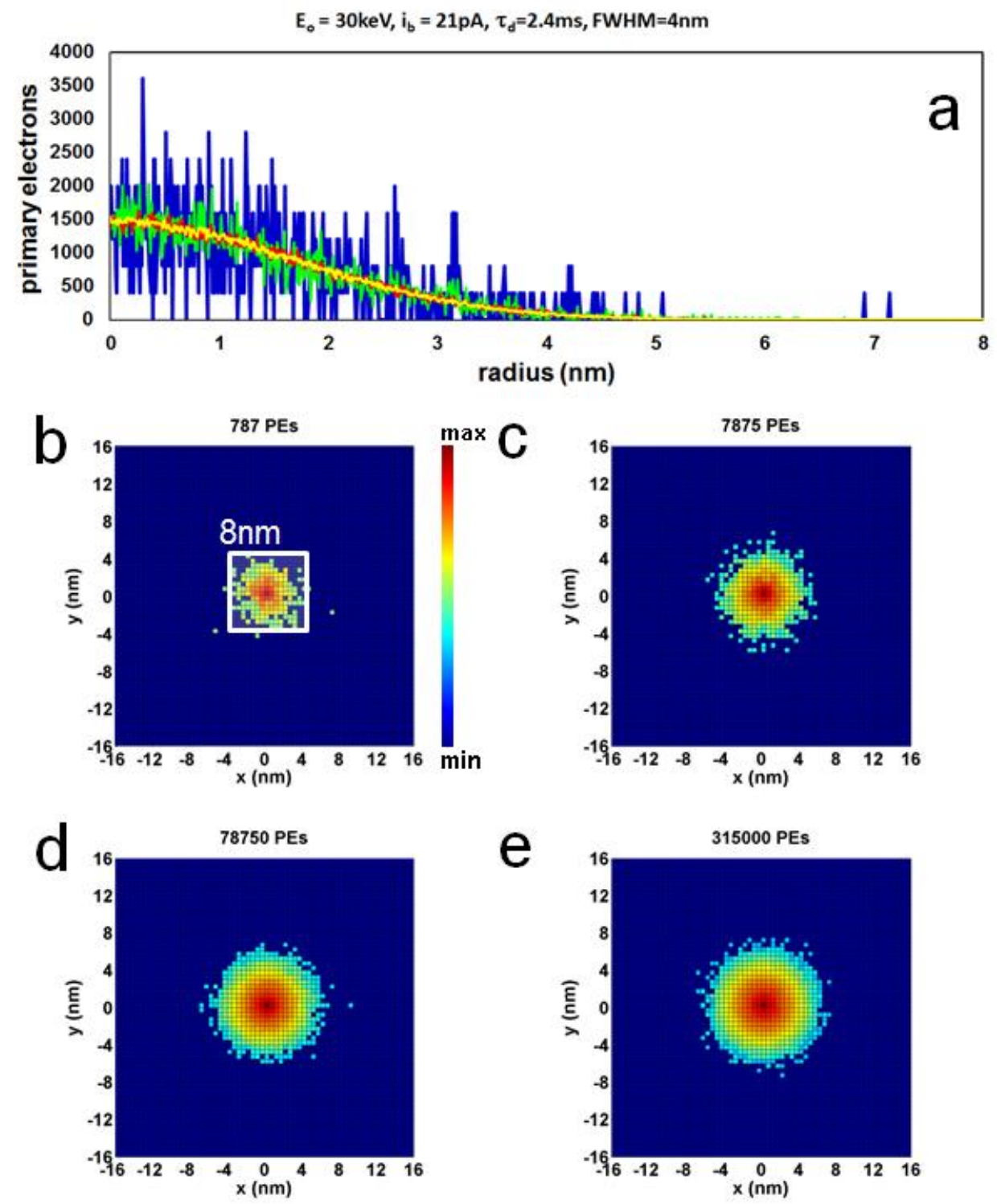

Figure S4.1 The radial Gaussian beam profile estimated for the case of a beam with a characteristic width of $\mathrm{FWHM}=4 \mathrm{~nm}$, a current of $21 \mathrm{pA}$ and beam dwell time of $2.4 \mathrm{~ms}$. This current and dwell time setting amounts to 315,000 incident electrons. The beam profile can be approximated by using only $0.25 \%$ (blue), $2.5 \%$ (green), $25 \%$ (red) and $100 \%$ (yellow) of the actual 315,000 electrons to construct the profile. Renormalization of each profile yields an integrated number of electrons for each profile of 315,000 . 


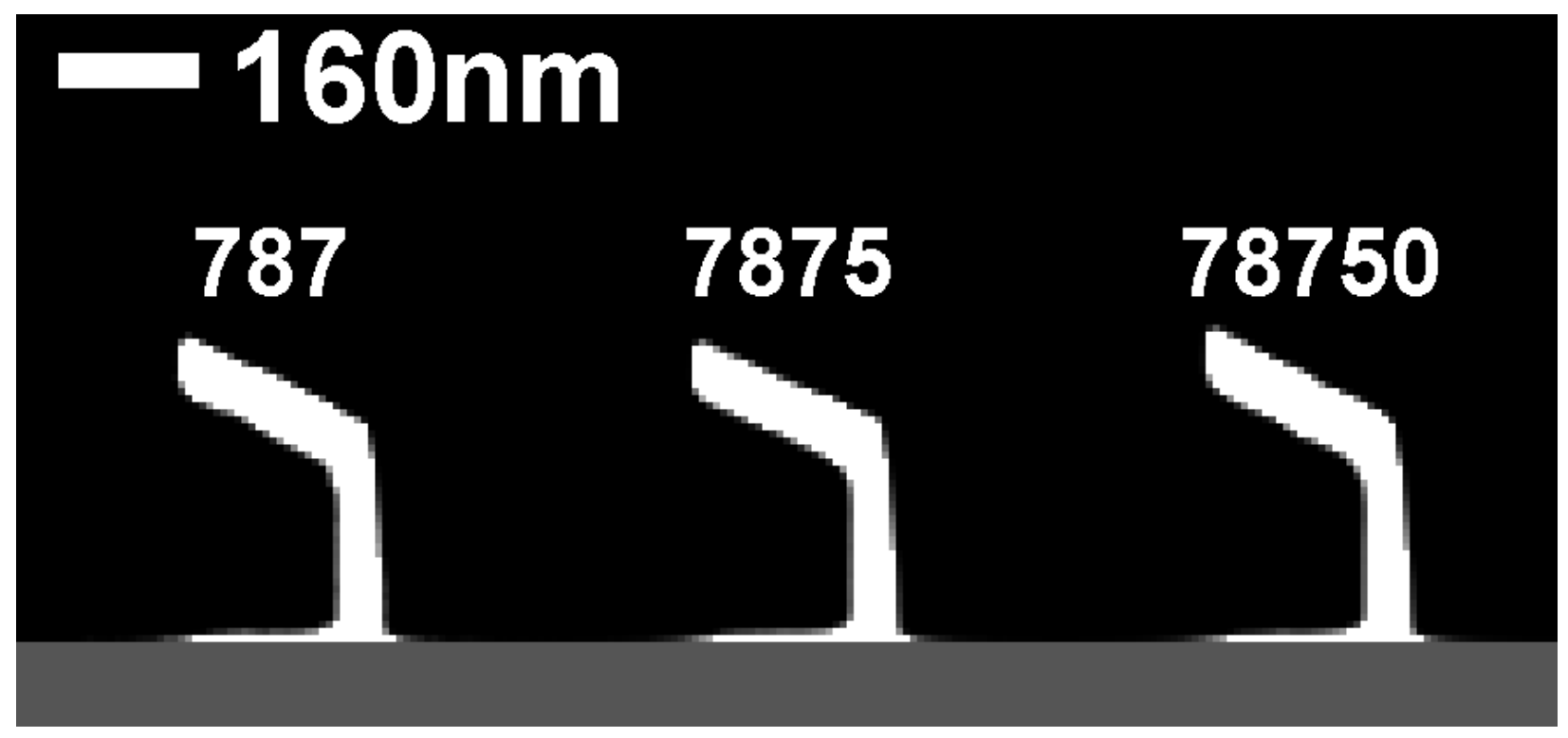

Figure S4.2 A FEBID segment constructed using a $2.4 \mathrm{~ms}$ dwell time per pixel. Number of electrons (superimposed) as few as 787 could be used to construct the beam profile without impacting simulation results, e.g., the segment angle is roughly the same for each of the three complementary shown. 


\section{Supplement 5}

There exist 9 unique surface voxel configurations, relative to the six, $1^{\text {st }}$ nearest neighbor voxels, in a 3D cubic lattice ${ }^{1}$. Five configurations dominate the surface area for a growing segment; classes 2-6 as provided by Mullikin and Verbeek ${ }^{2}$. The average surface area per voxel based on unbiased random plane orientations, assuming that each surface voxel type is equally probable in the segment geometry, yields an average, empty surface area $2.2 \Delta z^{2}$ per voxel. Surface voxels fill dynamically as deposition ensues from an empty surface area of $2.2 \Delta z^{2}$ to zero surface area. Thus, a further estimate of the average surface area taken over the filling process is simply $2.2 \Delta z^{2} / 2$, or $1.1 \Delta z^{2}$ which we assume is simply $\Delta z^{2}$.

\section{Supplement 6}

\section{Precursor Beam Influence \& Surface Normal Estimation}

A projected beam of precursor molecules introduces a directed component in the precursor flux which depends on the angle of incidence of the beam with respect to the surface normal for a given surface voxel ' $S$ '. The surface normal vector is thus estimated for each surface voxel contained inside the simulation domain as described below. Ultimately, the surface normal vector is used to calculate the number of impinging precursor molecules per surface voxel.

The calculation for the estimation of the surface normal vector uses the volume fraction of deposited solid contained within a $3 \times 3 \times 3$ 'cubic block' of voxels. The central voxel in this cubic block contains the surface voxel-of-interest (VOI) for calculation of the surface normal vector. The voxel edge length is $\Delta z=8 \mathrm{~nm}$. Thus, each cubic block has a $24 \mathrm{~nm}$ total edge length. Figure S6(a) shows a vertical slice of a representative cubic block which passes through the central surface voxel. In the following summary of the calculation, the 2D slice is used for clarity of demonstration, relative to a true 3D depiction.

The surface normal is calculated in part using the volume fraction (D) of deposit contained within each surface voxel contained in the cubic block. ' $D$ ' can be expressed as;

$$
D(\mathrm{x}, \mathrm{y}, \mathrm{z})=\frac{V(x, y, z)}{\Delta z^{3}}[1]
$$

where $V(x, y, z)$ is the total volume of deposit currently in the voxel, $\Delta z^{3}$ is the voxel volume. Thus, surface voxel occupancy has a possible range of;

$$
0 \leq D(x, y, z)<1
$$

Conversion of a ' $\mathrm{D}$ ' voxel into a bulk voxel ' $\mathrm{B}$ ' occurs when $\mathrm{D}=1$ and indicates complete filling. Any time that this condition is satisfied during the simulation, a sub-routine is triggered which updates the ' $\mathrm{S}$ ', 'B' and ' $\mathrm{V}$ ' status of the neighbor voxels. Voxels residing in the vapor phase are indicated by ' $\mathrm{V}$ '. 


\section{Deposition accumulates in surface voxels}

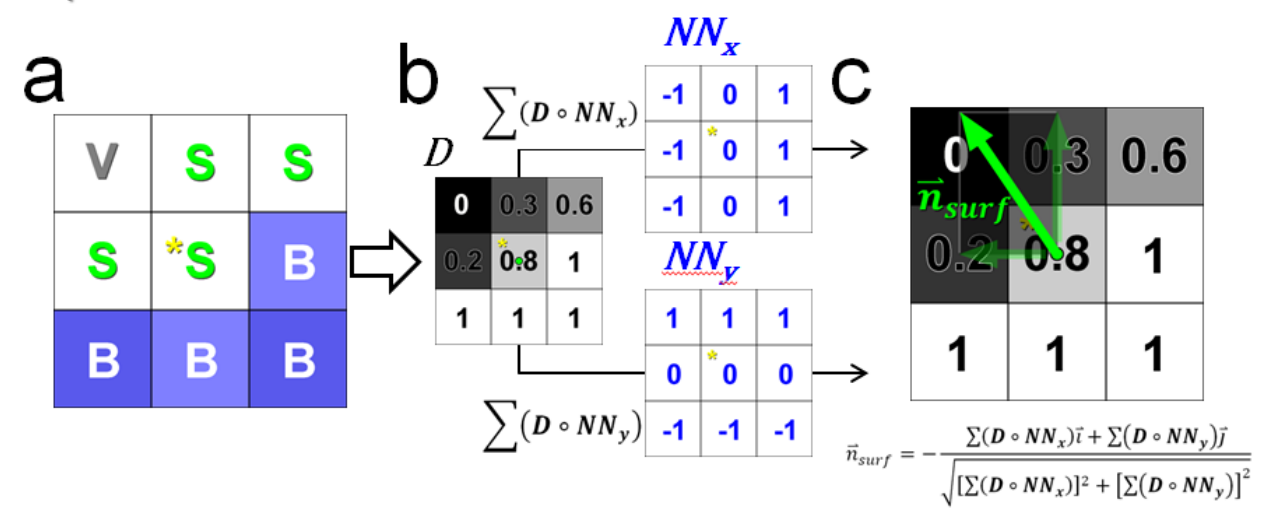

Specular precursor flux depends on the local surface normal

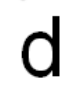

$$
\Phi_{T}=\Phi_{b}+\Phi_{\text {beam }} \frac{\vec{n}_{\text {surf }} \cdot\left(-\vec{n}_{\text {gas }}\right)}{\left|\vec{n}_{\text {surf }}\right|\left|\vec{n}_{\text {gas }}\right|}
$$

Figure S6 (a) A slice through a cubic block $(3 \times 3 \times 3)$ of voxels. 'V' indicates a vapor phase voxel, ' $S$ ' a surface voxel and 'B' a bulk voxel. The surface normal calculation, for the element of surface contained in the voxel labeled ' $S^{*}$ ', requires the volume fraction of deposit ' $D$ ' contained within each ' $S$ ' voxel in the $(3 \times 3 \times 3)$ cubic block. (In this figure, a $2 D$ representation of a $3 D$ calculation is shown for clarity) (b) A slice though the cubic block in (a) showing complementary example values for ' $D$ '. Elementwise multiplication of the cubic block ' $D$ ' with the nearestneighbor block in each of the three Cartesian coordinates makes it possible to estimate the surface normal vector according the equation shown in (c). (d) Ultimately, the precursor molecular flux $(\Phi)$ arriving at the voxel ' $S^{*}$ ' is the superposition of a diffuse component $\left(\Phi_{b}\right)$, which is independent of the surface normal vector, and a directed component $\left(\Phi_{\text {beam }}\right)$ which requires the estimated surface normal vector $\left(\mathbf{n}_{\text {surf }}\right)$.

The summation of the elementwise multiplication of ' $\mathrm{D}$ ' with a nearest neighbor array, i.e., $\mathrm{NN}_{\mathrm{x}}$, $\mathrm{NN}_{\mathrm{y}}$ and $\mathrm{NN}_{\mathrm{z}}$ shown in figure S6(b), for each Cartesian dimension yields an estimate of the surface normal (figure S6(b)) at the VOI. The unit surface normal is recovered according to the equation provided in figure S6(c) which is shown as the green vector - the $z$ and $x$ components are also shown in partial transparency.

The total precursor pressure per voxel is divided into both a directed component $\left(P_{\text {beam }}\right)$ based on the gas flux vector, and a diffuse (referred to a background $\mathrm{P}_{\mathrm{b}}$ ) component;

$$
P_{T}(x, y, z)=P_{b}+\operatorname{Shad}(x, y, z) \cdot P_{\text {beam }} \cdot \cos (\beta(x, y, z))
$$

$\operatorname{Shad}(x, y, z)$ is a logic variable $(0 / 1)$ that accounts for any shadowing that occurs from the FEBID deposit which can obstruct the line-of-sight of the precursor to the $\mathrm{VOI} \cos (\beta)$ takes into account the local orientation of the surface normal with respect to the gas impingement vector. The simulation requires input of $\mathrm{P}_{\mathrm{T}}$ and, $\mathrm{P}_{\mathrm{b}}$ and/or $\mathrm{P}_{\text {beam }}$. Importantly, $\mathrm{P}_{\text {beam }}$ is the maximum of 
the directed component of pressure. The purely diffuse partial pressure $\left(\mathrm{P}_{\mathrm{b}}\right)$ is a constant at all surface voxels in the simulation domain. The diffuse precursor flux is derived from the local pressure $(P)$ according to;

$$
\Phi=\frac{P}{\sqrt{M W \cdot T}}[4]
$$

where MW is the molecular weight of the precursor and $\mathrm{T}$ the precursor temperature. The total precursor flux impinging on the $\mathrm{VOI}$ is then;

$$
\Phi_{T}(x, y, z)=\Phi_{b}+\operatorname{Shad}(x, y, z) \cdot \Phi_{\text {beam }} \cdot \cos (\beta(x, y, z)) \quad[5]
$$

The directed component is treated as a function of the dot product between the surface normal ( $\left.\mathbf{n}_{\text {surf }}\right)$ at ' $\mathbf{S}^{* \text { ' }}$ and the (negative) of the gas propagation vector $\left(\mathbf{n}_{\text {gas }}\right)$. An arbitrary surface is generated in order to demonstrate how the directed component of the precursor flux is treated. The diffuse component was set to equal zero in case shown in figure 2(e) example. The colormap represents the surface height while the brightness of each pixel indicates the number of molecules impinging at the surface. The equation provided as figure $\mathrm{S} 6(\mathrm{~d})$ is valid from $\beta=$ $0-90^{\circ}$ where $\beta$ is the angle between $\mathbf{n}_{\text {surf }}$ and $-\mathbf{n}_{\text {gas }}$;

$$
-\left(\vec{n}_{\text {gas }}\right) \cdot \vec{n}_{\text {surf }}=\left|\vec{n}_{\text {gas }}\right|\left|\vec{n}_{\text {surf }}\right| \cos (\beta) \quad[6]
$$

The directed component of pressure is 0 for $\beta>90$ which we refer to as 'local' shadowing where the geometric cross-section for precursor impingement at that surface element is 0 . $A$ projection, ray casting scheme is implemented to determine 'remote shadowing' where precursor impingement is 0 due to a remote obstacle casting a shadow at the surface VOI. 


\section{Supplement 7}

The remaining calibration factor $(\chi)$ required scaling FEBID by 2.1 to achieve convergence between experiment and simulation. Even more recently, the pre-exponential variable and activation energy describing $\mathrm{MeCpPt}^{\mathrm{lV}} \mathrm{Me}_{3}$ physisorption have been determined and yield $\tau \sim 5$ ms for our RT growth conditions ${ }^{3}$. Precursor surface coverage increases with increasing $(\tau)$ leading to more dissociation according to the term $\sigma \cdot C \cdot i_{S E}$ in equation 3 main text. It was shown that a lower value of the scaling factor $(\chi)$ could be used $(\chi=1.5)$ in order to reproduce the experimental derived segment angle curve provided in figure 4(b) main text with very little change in segment object morphology, a further step towards convergence.

\section{Supplement 8}

In our previous paper we reported a diffusion coefficient of $0.65 \mu \mathrm{m}^{2} / \mathrm{s}$ for $\mathrm{MeCpPt}^{\mathrm{IV}} \mathrm{Me}_{3}$ diffusion on a $\mathrm{PtC}_{\mathrm{x}}$ surface ${ }^{4}$. Recently, a more refined value of $0.4 \mu \mathrm{m}^{2} / \mathrm{s}$ has been published ${ }^{5}$. The updated value was used here. Also in ${ }^{6}$, we used a total precursor pressure of 15 mTorr. In reality, this is a somewhat floating parameter due to the fact that (1) the sticking probability of $\mathrm{MeCpPt}^{\mathrm{IV}} \mathrm{Me}_{3}$ on $\mathrm{PtC}_{\mathrm{x}}$ is unknown (we assume $\delta=1$ here) and (2) the multiplication of the precursor pressure with the sticking probability $\left(\delta^{*} P_{\mathrm{T}}\right)$ dictates in the precursor surface accumulation term in the rate models making is difficult to isolate either value. Lastly, the surface density of the deposit has been updated from $9.6 \mathrm{PtC}_{5}$ molecules $/ \mathrm{nm}^{2}$ to $4.8 \mathrm{PtC}_{5}$ molecules $/ \mathrm{nm}^{2}$ which is more accurate estimate of deposit surface density based on the deposit density of $4.5 \mathrm{~g} / \mathrm{cm}^{3}$. 


\section{Supplement 9}

$\mathrm{PtC}_{5}$ thin film prisms (width $=500 \mathrm{~nm}$ ) were simulated as a function of the specular to diffuse pressure ratio. The purpose of the simulation set was to determine the ratio that simultaneously reproduced (1) the maximum height of the prism and (2) the ratio of minimum ledge height to the maximum ledge height derived from experiments.

The lateral extent of the beam exposure in the $(x-y)$ plane was $500 \mathrm{~nm} \times 500 \mathrm{~nm}$ for simulations while it was $2000 \mathrm{~nm} \times 2000 \mathrm{~nm}$ for real experiments. The total $\mathrm{MeCpPt}{ }^{1 \mathrm{~V}} \mathrm{Me}_{3}$ pressure was fixed at $30 \mathrm{mT}$ Trr while the specular/diffuse (in units of mTorr/mTorr) components varied over the range $30 / 0,25 / 5,20 / 10,15 / 15,10 / 205 / 25$. The beam acceleration voltage was $5 \mathrm{keV}$, the primary beam current was $400 \mathrm{pA}$ and the beam size was $\mathrm{FWHM}=27 \mathrm{~nm}$. The spiral-out beam scanning strategy, shown in figure 2(a), main text, was used where the pattern rotation angle $(\alpha)$ was $15^{\circ}$.

Simulation results are presented as height histograms in figure S9(a) along with the experimental results (black histogram). The collection of histograms have been shifted along the $y$-axis for both clarity and comparison purposes. The experimental value for ratio representing the minimum ledge height to maximum ledge height was 0.68 for the experiment and is superimposed on the histogram. Also, the experimental histogram has been extended down the figure as a shaded flow in order to compare the results with simulations. The actual AFM tapping mode height image is shown in figure S9(b) with the ledge of minimum height ' 3 ' and the ledge of maximum height labelled ' 1 '.

The simulation at 20 mTorr specular pressure and 10 mTorr diffuse pressure (blue curve) best represented the experimental result (while also later describing results at $30 \mathrm{keV}$ for the $3 \mathrm{D}$ segment based objects) in terms of the ledge surface morphology, ledge height ratio $(=0.65)$ and only slightly under represented the maximum height by $5 \mathrm{~nm}$. Complementary, simulated morphologies are shown in figure S9(c) where the color border around the images matches the histogram in figure S9(a). At higher diffuse pressures, the maximum height better reflected experiments but at the expense of the ledge morphology which became more uniform across the prism (see red and orange histogram complementary images in figure S9(c)). 


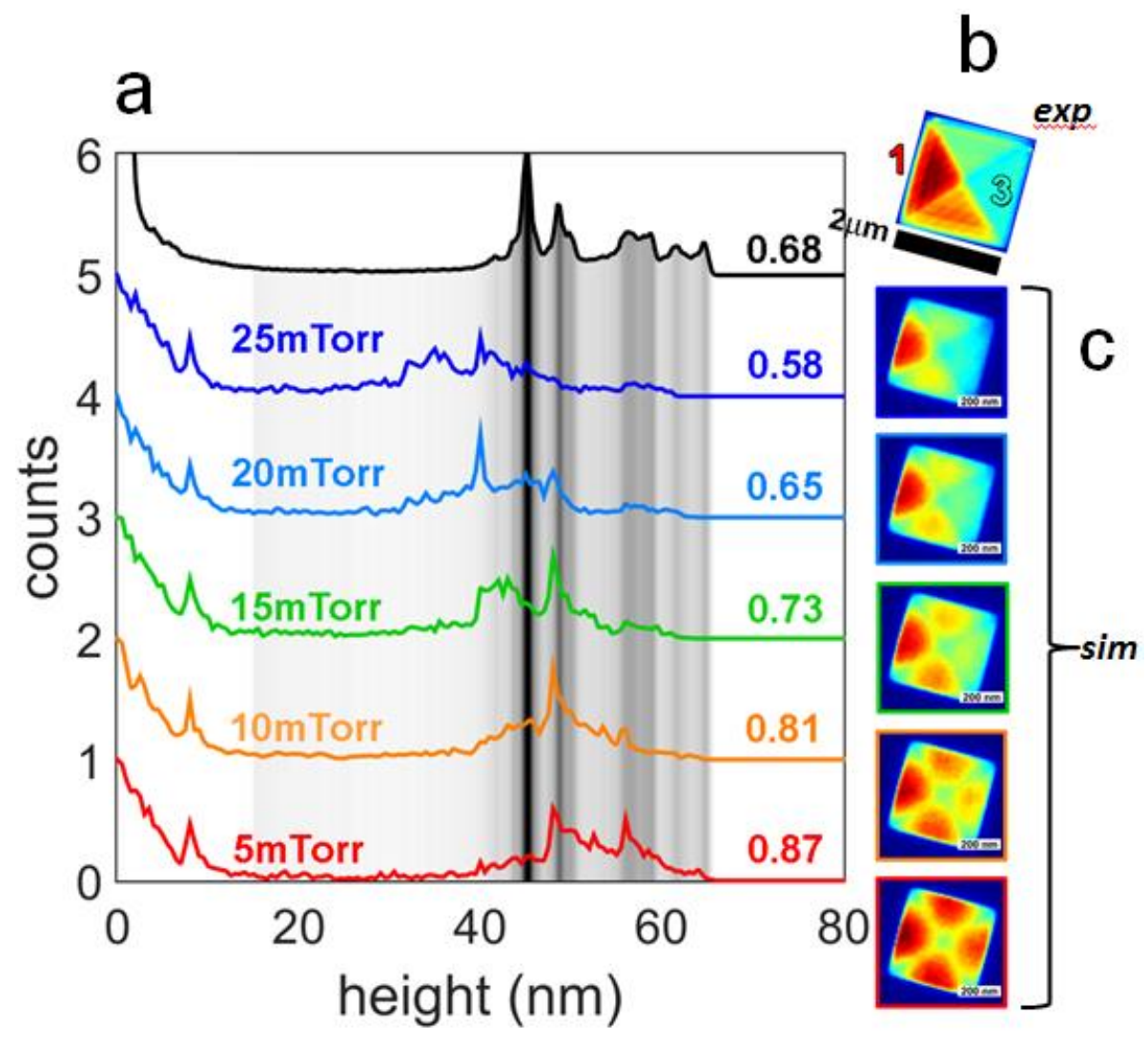

Figure S9 (a) Height histograms for thin film prisms prepared by FEBID using the spiral-out scanning strategy. Each individual histogram has been shifted in the counts coordinate to avoid overlap and provide visual clarity. The black histogram was derived from atomic force microscopy data (b). The value of 0.68 associated with the black histogram is the height ratio calculated by (b) dividing the mean height of the lowest ledge (3) on the thin film prism surface by the mean height of the highest ledge (1). The remaining simulations are complementary simulations of the experiment where the ratio of diffuse-to-directed pressure was varied, at constant total pressure, in order to the replicate the experimental ledge height ratio. The directed pressure of 20 mTorr and background pressure of $10 \mathrm{mTorr}$ best reproduced the results. 


\section{Supplement 10}

The ability to collect the sample current signal from the focused electron beam induced deposition (FEBID) simulation was tested in response to a reviewer request. The test consisted of simulating FEBID for a published morphology, a so-called "tip on suspended rod" structure, which included a sample current trace acquired during the growth of the structure (figure S10(a)). The published information can be found in, "Electron range effects in focused electron beam induced deposition of 3D nanostructures", Microelectronic Engineering 83, 1482-1486 (2006) on page 1485 in figure 4. The "tip on suspended rod" was grown in experiments using an acceleration voltage of $25 \mathrm{keV}$, a beam current of $500 \mathrm{pA}$ and a beam width of $4 \sigma \sim 132 \mathrm{~nm}$ using the precursor hexafluoro-acetylacetonato-copper(I)-vinyltrimethylsilane. In the EBID simulation (figure $S 10(b)$ ), an acceleration voltage of $30 \mathrm{keV}$, a beam current of $21 \mathrm{pA}$ and a beam width of $\mathrm{FWHM}=5 \mathrm{~nm}$ using the precursor $\mathrm{MeCp}\left(\mathrm{Pt}^{\mathrm{IV}}\right) \mathrm{CpMe}_{3}$. The precursor $\mathrm{MeCp}\left(\mathrm{Pt}^{\mathrm{IV}}\right) \mathrm{CpMe}_{3}$ was used in place of (hfac)CuVTMS because fewer parameters are currently available for the hfac(Cu)VTMS precursor. Moreover, a smaller version of the "tip on suspended rod" morphology was simulated in order in reduce the total simulation process time.
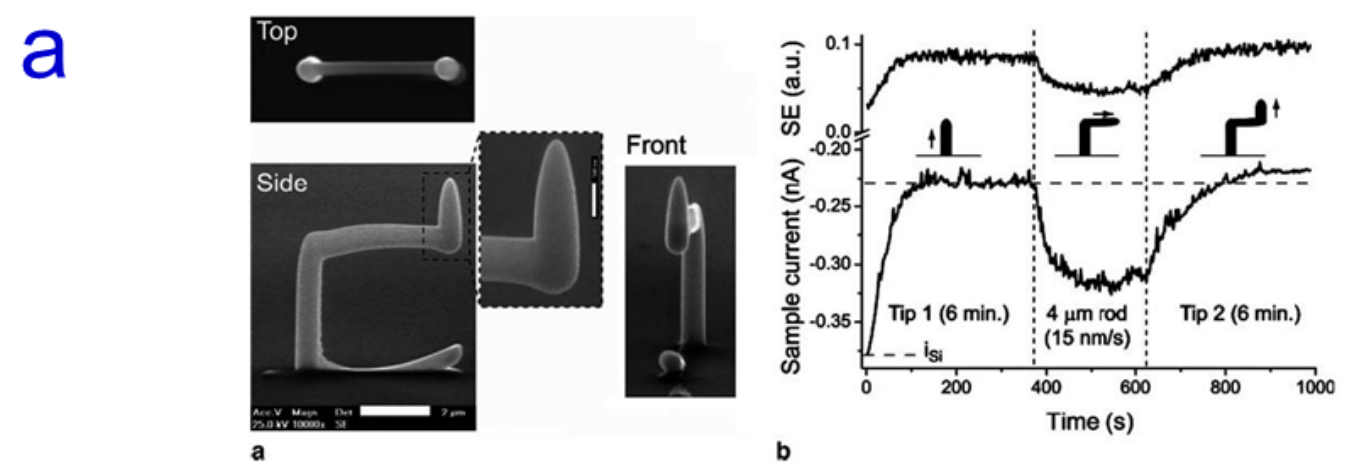

Reproduced with permission from, Bret, T.; I. Utke; Hoffmann, P.; Abourida, M.; Doppelt, P.; "Electron range effects in focused electron beam induced deposition of 3D nanostructures", Microelectronic Engineering 2006, 83, 1482-1486. This image appeared as figure 4 .
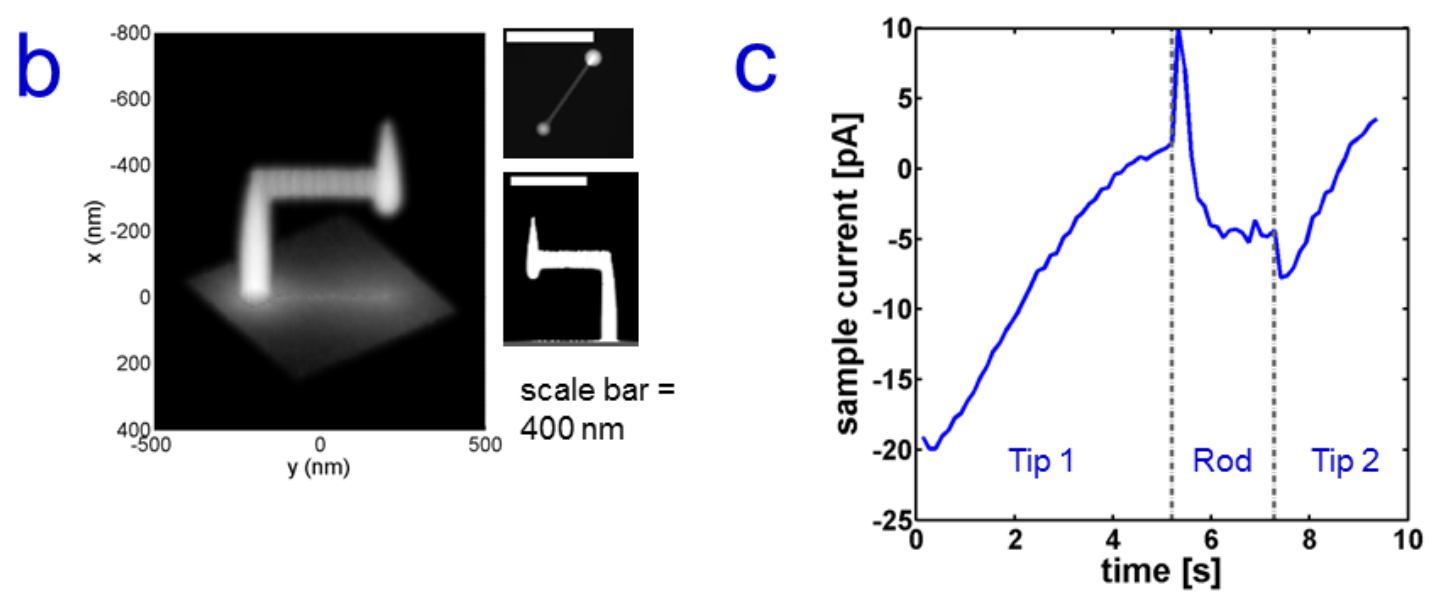

Figure S10 (a) The "tip on suspended rod" structure grown using an acceleration voltage of 25 $\mathrm{keV}$, a primary electron beam current of $500 \mathrm{pA}$ and the precursor hexafluoro-acetylacetonato- 
copper(I)-vinyltrimethylsilane". (b) A 3D FEBID simulation of the "tip on suspended rod" structure using an acceleration voltage of $30 \mathrm{keV}$, a primary electron beam current of $21 \mathrm{pA}$ and the precursor $\mathrm{MeCp}\left(\mathrm{Pt}^{\mathrm{IV}}\right) \mathrm{CpMe}_{3}$. (c) The stage sample current acquired during the 3D FEBID simulation. Compare with the stage current collected experimentally in (b).

The acceleration voltage, in part, determines the sample current signal because the penetration depth of the primary electrons is very large (tens of micrometers at $25-30 \mathrm{keV}$ ) relative to the tip and rod dimensions (see S2). In addition, although the deposits consist of different metals, (1) carbon contamination contributes significantly to the composition ( 55 at\% $\mathrm{C}$ for the $\mathrm{Cu}$ experiment and $\sim 83$ at\% $\mathrm{C}$ for the Pt simulation) making the relative material parameters such as atomic number, molecular weight and density, which dictate beam penetration, comparable.

The secondary electrons that are liberated from the deposit when the primary beam intersects the surface contribute significantly to the sample current signal, along with primary electrons that penetrate and dissolve in the solid. Primary electrons can also be scattered at very high angles away from the deposit due to large angle elastic scattering although this is relatively low probability event relative to the other current affecting factors.

The stage current signal can be collected periodically during FEBID and provides a reliable method to track changes in the deposit morphology, indirectly, without the use of secondary electron imaging. The current signal is described by;

$$
i_{s}=i_{b}-\delta_{I} i_{b}-\eta i_{b}-\delta_{I I} \eta i_{b} \quad[1]
$$

where $\left(i_{s}\right)$ is the sample current, $\left(i_{b}\right)$ is the incident primary beam current, $\left(\delta_{l}\right)$ is the secondary electron number 1 yield, $\left(\delta_{\| I}\right)$ is the secondary electron number 2 yield and $(\eta)$ is the backscattered electron yield. The total secondary electron yield $\left(\delta_{\mathrm{T}}\right)$ is given by;

$$
\delta_{T}=\delta_{I}+\delta_{I I} \eta[2]
$$

The continuum simulation domain boundary is typically $1-2 \mu \mathrm{m}$ in edge length along the $\mathrm{x}, \mathrm{y}$ and $z$ dimensions. This has the effect of discounting the terms $\left(\eta \mathrm{i}_{b}\right)$ and $\left(\delta_{\|} \eta \mathrm{i}_{\mathrm{b}}\right)$ because the backscattered electrons fall outside the simulation region. However, due to the deep beam penetration at 25 and $30 \mathrm{keV}$, described above, these terms contribute relatively little compared with $\left(i_{b}\right)$ and $\left(\delta_{l}\right)$. Thus, the sample current derived from the simulation (figure $S 10(c)$ ) resembled closely the experimental curve. Specifically, the sample current shape during the growth of the (1) tip, (2) rod and (3) tip geometries was the same for both experiments and simulations. The only noticeable differences are the spikes observed in the simulated sample current at the tip/rod and rod/tip boundaries. This observed difference is attributed to the difference in the beam pixel point pitch $(\Lambda)$ to beam diameter (FWHM) ratio between experiments $(\Lambda / F W H M=0.48 \mathrm{~nm} / 55 \mathrm{~nm}=0.009)$ and simulation $\left(\Lambda / 2 r_{b}=1 \mathrm{~nm} / 4 \mathrm{~nm}=0.25\right)$. For example, when the beam displacement begins after the growth of the first tip the beam makes a relatively large displacement relative to the tip diameter striking the highly tapered apex causing a large ejection of secondary electrons to the large surface area exposed to the beam. 
EBID Simulation growth conditions

An acceleration voltage of $30 \mathrm{keV}$ and a beam current of $21 \mathrm{pA}$ used to simulate the FEBID of the "tip on suspended rod". The beam radius was $4 \mathrm{~nm}$, the beam dwell time per pixel was $5.2 \mathrm{~ms}$ and the pixel point pitch during the rod growth was $1 \mathrm{~nm}$. A total of 1000 stationary beam dwells were used to grow the first tip. Next, the rod was deposited with 400 beam dwells at a beam speed of $(v=192 \mathrm{~nm} / \mathrm{s})$. The final tip was deposited also using 400 beam dwells. The total pressure was $30 \mathrm{mTorr} \mathrm{MeCp}\left(\mathrm{Pt}^{\mathrm{IV}}\right) \mathrm{CpMe}_{3}$. 


\section{Supplement 11}
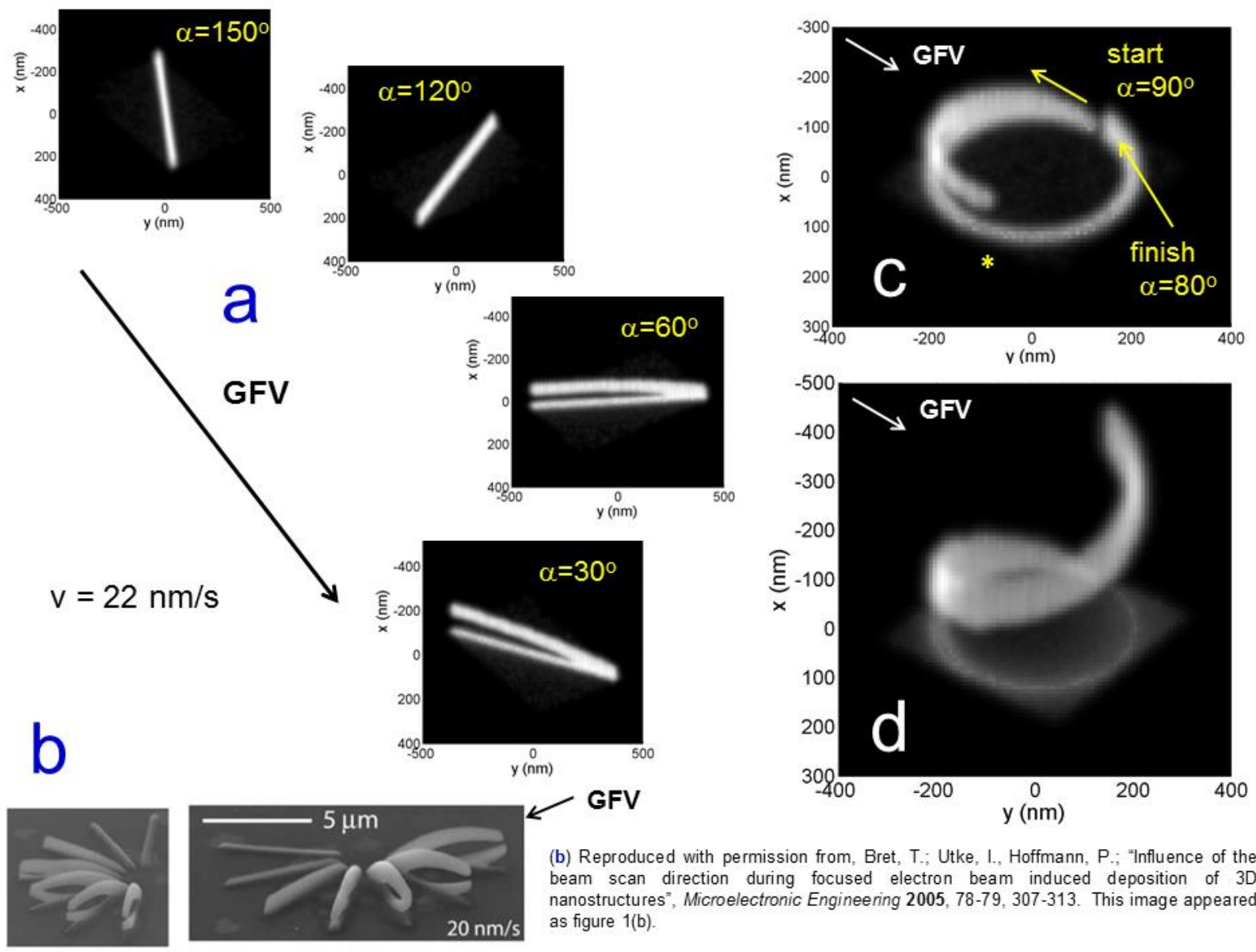

GFV

(b) Reproduced with permission from, Bret, T.; Utke, I., Hoffmann, P.; "Influence of the beam scan direction during focused electron beam induced deposition of 3D nanostructures", Microelectronic Engineering 2005, 78-79, 307-313. This image appeared as figure $1(\mathrm{~b})$.

Figure 11 (a) Virtual SEM images (tilt $=52^{\circ}$ ) of four different 3D FEBID $\mathrm{MeCp}\left(\mathrm{Pt}^{\mathrm{IV}}\right) \mathrm{CpMe}_{3}$ simulations with a variable electron beam scanning directions. Each scan was an $800 \mathrm{~nm}$ line scan with a pixel point pitch of $0.53 \mathrm{~nm}$ and a constant electron beam velocity of $22 \mathrm{~nm} / \mathrm{s}$. (b) (hfac)CuVTMS experiments reported $\mathrm{in}^{8}$. The direction of the gas flux vector (GFV) during the experiments is superimposed for reference. (c) A virtual SEM image (tilt $=38^{\circ}$ ) of a circular beam scanning pattern. The radius of the pattern was $200 \mathrm{~nm}$. The precursor flux was $66 \%$ directed and $33 \%$ diffuse during the simulation for a total pressure of $1.3 \mathrm{mTorr}$ $\mathrm{MeCp}\left(\mathrm{Pt}^{\mathrm{IV}}\right) \mathrm{CpMe}_{3}$. (d) The results of a complementary simulation to (c) where the directed component of the precursor flux is $0 \%$ and the diffuse component is $100 \%$, again for a total pressure of 1.3 mTorr.

In the article ${ }^{8}$, Bret et al., "Influence of the beam scan direction during focused electron beam induced deposition of 3D nanostructures", Microelectronic Engineering 78-79, 307-313 (2005), the direction of gas nozzle was shown to strongly influence the vertical growth rate depending on the scanning during FEBID, more so than was observed during the simulations and experiments reported in this article. However, the strong GFV dependence was observed in 
simulations when the beam speed, electron dose and precursor surface coverage were adjusted to match the experiments reported ${ }^{8}$.

The simulations reported here have implemented a primary beam current of $21 \mathrm{pA}$ and a beam size of FWHM $=4 \mathrm{~nm}$. Conversely, a beam current of $500 \mathrm{pA}$ and a beam diameter of $55 \mathrm{~nm}$ was used $\mathrm{in}^{8}$. A smaller total segment size was simulated in order in reduce the total simulation process time. Therefore, the simulated current density is;

$$
\left.e_{d}^{-}\right|_{\operatorname{sim}}=\frac{i_{b}}{\pi \cdot H W H M^{2}}=\frac{21[p A]}{\pi(2[n m])^{2}}=1.7\left[\frac{p A}{n m^{2}}\right]
$$

A representative experimental current density reported in ${ }^{8}$ was;

$$
\left.e_{d}^{-}\right|_{\exp }=\frac{i_{b}}{\pi \cdot H W H M^{2}}=\frac{500[p A]}{\pi(27[n m])^{2}}=0.2\left[\frac{p A}{n m^{2}}\right]
$$

In order to have an equivalent current density between experiments and simulations, a new simulation beam size was calculated using the reported electron current density $0.2 \mathrm{pA} / \mathrm{nm}^{2}$ yet keeping constant the primary beam current at $21 \mathrm{pA}$;

$$
H W H M=\sqrt{\frac{i_{b}}{\left.\pi e_{d}^{-}\right|_{\text {exp }}}}=5.5[\mathrm{~nm}]
$$

The beam dwell time per pixel used in the experiments $\left(\tau_{d}=24 \mathrm{~ms}\right)$ was also used in the simulation to achieve a common primary electron dose;

$$
\frac{i_{b} \tau_{d}}{\pi \cdot H W H M^{2}}=\frac{21[p A] \cdot 24 \times 10^{-3}[s]}{\pi(5.5[n m])^{2}}=5.3 \times 10^{-3}\left[\frac{p C}{n^{2}}\right]
$$

In order to further close the gap between the experiments and simulations, the equilibrium coverage of (hfac)CuVTMS reported in ${ }^{8}$, of $\sim 10^{-1}$ (hfac)CuVTMS molecules $/ \mathrm{nm}^{2}$, was adapted to the simulation by forcing the equilibrium precursor coverage of $\mathrm{Me}\left(\mathrm{Pt}^{\mathrm{IV}}\right) \mathrm{CpMe}_{3}$ to this value by changing the total pressure which is an input parameter to the simulation (a direct comparison between the two cases was not possible as the total electron impact dissociation cross section for hexafluoro-acetylacetonato-copper(I)-vinyltrimethylsilane is unknown). Based on the reported value of 0.1 molecules $/ \mathrm{nm}^{2}$, the precursor site density of $2.8 \mathrm{Me}\left(\mathrm{Pt}{ }^{\mathrm{IV}}\right) \mathrm{CpMe}_{3}$ sites $/ \mathrm{nm}^{2}$ yields a fractional surface coverage $(\theta)$ of $\sim 0.03$, or $3 \%$ coverage. However, initial simulations revealed that the segment geometry does not lift-off of the substrate surface vertically as observed in experiments (discussed below). In order to achieve substrate lift-off, the total pressure was raised from $\sim 0.4 \mathrm{mTorr}$ to $\mathrm{P}_{\mathrm{T}}=1.3 \mathrm{mTorr}$ (where the directed component $\left(P_{\text {beam }}\right.$ ) of $0.875 \mathrm{mTorr}$ and a background pressure $\left(P_{b}\right)$ of $0.417 \mathrm{mTorr}$ ) yielding $10 \%$ surface coverage of $\mathrm{Me}\left(\mathrm{Pt}^{\mathrm{IV}}\right) \mathrm{CpMe}_{3}$. Complementary experimental and simulated results were achieved under these conditions.

$3 D$ EBID simulation growth conditions 
An acceleration voltage of $30 \mathrm{keV}$ and a beam current of $21 \mathrm{pA}$ used to simulate the FEBID of the low angle segment features. The beam radius was $5.5 \mathrm{~nm}$, the beam dwell time per pixel was $24 \mathrm{~ms}$ and the pixel point pitch during the rod growth was $0.53 \mathrm{~nm}$. The total length of the segments grown was $800 \mathrm{~nm}$. The beam velocity was $v=0.53 \mathrm{~nm} / 0.024 \mathrm{~s}=22 \mathrm{~nm} / \mathrm{s}$.

Figure S11(a) shows the results of four FEBID simulations where the beam scanning direction was changed relative to the gas flux vector (GFV). The superimposed angle-based values provided in figure $\mathrm{S} 11$ (a) represent the orientation of the beam scanning direction with respect to the GFV. For example, a value of $180^{\circ}$ indicates the beam is scanning away from the GFV, parallel to the projection of the GFV in the substrate plane. Conversely, a value of $0^{\circ}$ indicates anti-parallel scanning where the beam is moving toward the GFV. This is the most favorable condition for vertical growth. This angle is referred to as the rotation angle $(\alpha)$ in the main text.

\section{Similarities between experiments and simulations}

Several similarities were found between the published (hfac)CuVTMS experiments and the $\mathrm{Me}\left(\mathrm{Pt}^{\mathrm{lV}}\right) \mathrm{CpMe}_{3}$ simulations. The comparison is made for a common beam speed; $(\mathrm{v}=22 \mathrm{~nm} / \mathrm{s}$ simulations, figure $S 11(\mathrm{a}))$ and $(\mathrm{v}=20 \mathrm{~nm} / \mathrm{s}$ experiments, figure $\mathrm{S} 11(\mathrm{~b}))$. A strong dependence of the vertical growth rate on the GFV orientation was observed in both cases: nanowires are grown only on the surface in the range of $\alpha=90^{\circ}-180^{\circ}$ while self-suspended segments are grown for $\alpha=0^{\circ}-90^{\circ}$. The GFV vector in figure $S 11$ (b) indicates $\alpha=0^{\circ}$, or the direction of antiparallel scanning for the experiments. In the case of segment growth, an underlying nanowire is produced underneath the segment for both cases. EBID simulations also revealed that the nanowire height increased with a decrease in $(\alpha)$ as seen clearly in the experiments (figure $S 11$ (b) In figure $S 11$ (a) the nanowire labelled $\left(\alpha=150^{\circ}\right.$ ) was $55 \mathrm{~nm}$ thick while the nanowire at $\left(\alpha=120^{\circ}\right)$ was $70 \mathrm{~nm}$ thick.

Ultimately, the reduction in surface coverage from $74 \%$ to $10 \%$ was required to reproduce the GFV-beam scanning dependence for a comparable current and linear dose rates between experiments and simulations. Nonetheless, because not all parameters are known for the (hfac)CuVTMS precursor the presented simulation results are circumstantial.

\section{Differences between experiments and simulations}

Experiments performed by Bret et al. ${ }^{8}$ revealed an underlying, secondary nanowire forms during FEBID and can lift-off of the substrate surface while the initial, suspended segment is still growing above $(20 \mathrm{~nm} / \mathrm{s}$ panel in figure $S 11(b))$. This was not observed in simulations. In simulations it was found that the height of the underlying nanowire was relatively smaller and would only lift-off the substrate in cases where the beam velocity exceeded the lateral growth velocity of the segment causing the beam to fall off the segment starting the growth of a new nanowire. The observed difference in the underlying nanowire growth could be due to the fact that kinetic growth conditions are not quite equivalent between the new cases. This discrepancy will be investigated further in the future. 
A circular scanning pattern makes it possible to investigate the GFV influence dynamically because the patterning orientation is continuously changing in a controlled and predictable fashion. Figure S11(c) shows a virtual SEM image of a 3D FEBID simulation where a circular scanning pattern was used. Beam scanning initiated at $\left(\alpha=0^{\circ}\right)$ indicated as the "starting" position in the virtual SEM image. This is the preferred orientation for lift-off. Vertical growth continues but continuously decreases as the beam turns continuously away from the gas nozzle. Eventually the beam velocity exceeds the lateral segment growth rate and the beam falls off of the segment. FEBID begins anew on the substrate surface and a new nanowire begins to thicken. FEBID was executed for $\Delta \alpha=350^{\circ}$.

An additional simulation was executed to determine the influence of the directed nature of the GFV. The simulation shown in figure $\mathrm{S11}$ (c) was repeated using the same total pressure of precursor $\left(\mathrm{P}_{\mathrm{T}}=1.3 \mathrm{mT}\right.$ Torr) but with a zero directed component of precursor (totally diffuse gas flux). Every voxel in the domain experienced the same molecular flux regardless of the orientation of the deposit surface. A complete, single turn helix can be deposited under these modified conditions figure S11(d) and provides a direct measure of the influence of the GFV, in this case its absence. 


\section{Supplement 12}

\section{S12_BeamDwellPattern.wmv}

\section{Supplement 13}

In this example, the surface voxels ' $S$ ' are rendered with a colormap that is linked to the $x$ direction. All other settings are the same as described in SI1.

\section{S13_Frame3DFEBID.wmv}




\section{Supplement 14}

Virtual SEM images are generated by projecting the 3D object on a virtual, flat rectangular screen. The screen position is characterized by an azimuthal, rotation and distance coordinate. The collection of information of the 'screen' empirically mimics real secondary electron collection at a very high positive collection bias such that all secondary electrons are captured. For example, in a real detector a certain fraction of SEs emitted, from the surface, with an unfavorable trajectory for collection may be reoriented at relatively high bias and collected, even if they are emitted from region lying in the shadow of the detector. The virtual screen had a pixel size equal to that of the simulation domain $=8 \mathrm{~nm}$. The 'intensity' contributed by a surface voxel to the screen was set equal to the fraction of deposit residing there. The position on the screen of the 'counts' was determined by projecting a vector from the deposit, to the screen, with an orientation parallel to the surface normal of the screen which is directed at typically the center-of-mass of the deposit. Even surface sites lying in the shadow of the detector, cast by the detector, were considered as collected mimicking the high bias condition mentioned above. The 'signal' was integrated without a possibility of saturation in counts and stored in the array $S E_{i m g}$. Image processing led to the final image intensity described by;

$$
S E_{i m g}\left(x_{s}, y_{s}\right)=\sqrt[3]{\int_{t} D_{i m g} \nabla^{2} S E_{i m g}\left(x_{S}, y_{s}\right) d t+\frac{S E_{i m g}\left(x_{s}, y_{s}\right)}{N_{c}}}
$$

which, again, is a purely empirical expression. Coordinates in the reference frame of the screen are $x_{s}$ and $y_{s}$ where $x_{s}(x, y, z)$ and $y_{s}(x, y, z)$. Qualitatively, the expression can be understood as follows. The $1^{\text {st }}$ term describes an image diffusion operation executed in the image plane to smoothen out frequencies imposed on the image by the voxel nature of the deposit. The $2^{\text {nd }}$ term averages the raw signal based on the number of counts which makes it possible to dampen the image contrast. Lastly, the $3^{\text {rd }}$ root makes it possible to image both the 3D object as well as the faint amount of substrate deposition due to the beam proximity effect.

\section{S14_Frame3DFEBID_vSEM.wmv}




\section{Supplement 15}

The cube edge length for these FEBID cube structures was $200 \mathrm{~nm}$ while the images shown in Figures $5 \mathrm{a}-\mathrm{b}$, in the main text, were grown with an cube edge length of $250 \mathrm{~nm}$.

\section{S15_Cube3DFEBID.wmv}

\section{S15_Cube3DFEBID_vSEM.wmv}

\section{Supplement 16}

\begin{tabular}{|l|l|l|l|l|}
\hline $\begin{array}{l}\text { Experiment / } \\
\text { machine }\end{array}$ & Precursor & $\begin{array}{l}\text { GIS angle to } \\
\text { substrate }\end{array}$ & $\begin{array}{l}\text { Vertical GIS } \\
\text { distance to } \\
\text { substrate }\end{array}$ & $\begin{array}{l}\text { Lateral GIS position to } \\
\text { beam center (X/Y) }\end{array}$ \\
\hline Pads/Nova 200 & $\mathrm{MeCpPt}^{\mathrm{IV}} \mathrm{Me}_{3}$ & $52^{\circ}$ & $180 \pm 20 \mu \mathrm{m}$ & $200 \pm 10 \mu \mathrm{m} / 40 \pm 10 \mu \mathrm{m}$ \\
\hline 3D / Nova 600 & $\mathrm{MeCpPt}^{\mathrm{V}} \mathrm{Me}_{3}$ & $52^{\circ}$ & $100 \pm 20 \mu \mathrm{m}$ & $0 \pm 10 \mu \mathrm{m} / 150 \pm 10 \mu \mathrm{m}$ \\
\hline 3D / Nova 200 & $\mathrm{MeCpPt}^{\mathrm{IV}} \mathrm{Me}_{3}$ & $38^{\circ}$ & $470 \pm 20 \mu \mathrm{m}$ & $30 \pm 10 \mu \mathrm{m} / 170 \pm 10 \mu \mathrm{m}$ \\
\hline 3D / Nova 200 & $\mathrm{Me}_{2} \mathrm{Au}(\mathrm{acac})$ & $52^{\circ}$ & $380 \pm 20 \mu \mathrm{m}$ & $40 \pm 10 \mu \mathrm{m} / 130 \pm 10 \mu \mathrm{m}$ \\
\hline 3D/ Nova 600 & $\mathrm{W}(\mathrm{CO})_{6}$ & $38^{\circ}$ & $100 \pm 20 \mu \mathrm{m}$ & $0 \pm 10 \mu \mathrm{m} / 150 \pm 10 \mu \mathrm{m}$ \\
\hline
\end{tabular}




\section{References (Supplement)}

1. Windreich, G.; Kiryati, N.; Lohmann, G. Voxel-based Surface Area Estimation: from Theory to Practice. Pattern Recogn. 2003, 36, 2531-2541.

2. Mullikin, J. C.; Verbeek, P. W. Surface Area Estimation of Digitized Plane. Bioimaging 1993, 1, 616.

3. Cullen, J.; Bahm, A.; Lobo, C. J.; Ford, M. J.; Toth, M. Localized Probing of Gas Molecule Adsorption Energies and Desorption Attempt Frequencies. J. Phys. Chem. C 2015, 119, 15948-15953.

4. Winkler, R.; Fowlkes, J.; Szkudlarek, A.; Utke, I.; Rack, P. D.; Plank, H. The Nanoscale Implications of a Molecular Gas Beam during Electron Beam Induced Deposition. ACS Appl. Mater. Interfaces 2014, 6, 2987-2995.

5. Cullen, J.; Lobo, C. J.; Ford, M. J.; Toth, M. Electron-Beam-Induced Deposition as a Technique for Analysis of Precursor Molecule Diffusion Barriers and Prefactors. ACS Appl. Mater. Interfaces 2015, 7, 21408-21415.

6. Winkler, R.; Geier, B.; Plank, H. Spatial chemistry evolution during focused electron beaminduced deposition: origins and workarounds. Appl. Phys. A: Mater. Sci. Process. 2014, 117, 1675-1688.

7. Bret, T.; Utke, I.; Hoffmann, P.; Abourida, M.; Doppelt, P. Electron Range Effects in Focused Electron Beam Induced Deposition of 3D Nanostructures. Microelectron. Eng. 2006, 83, 1482-1486.

8. Bret, T.; Utke, I.; Hoffmann, P. Influence of the Beam Scan Direction during Focused Electron Beam Induced Deposition of 3D Nanostructures. Microelectron. Eng. 2005, 78-79, 307-313. 